\title{
Agronomic characterization of high-yielding irrigated barley accessions in the Cerrado
}

\author{
Ricardo Meneses Sayd(1), Renato Fernando Amabile( ${ }^{(2)}$, Fábio Gelape Faleiro(2), \\ Ana Paula Leite Montalvão(1) and Mateus Costa Coelho(1)
}

\begin{abstract}
(1)Universidade de Brasília, Faculdade de Agronomia e Medicina Veterinária, Campus Universitário Darcy Ribeiro, Instituto Central de Ciências, Ala Sul, Caixa Postal 04508, CEP 70910-900 Brasília, DF, Brazil. E-mail: ricardo_sayd@hotmail.com, anapaulalmbrbsb@gmail.com, ccoelhomateus@gmail.com (2)Embrapa Cerrados, BR-020, Km 18, Caixa Postal 08223, CEP 73310-970 Planaltina, DF, Brazil. E-mail: renato.amabile@embrapa.br, fabio.faleiro@embrapa.br
\end{abstract}

Abstract - The objective of this work was to estimate the genetic, phenotypic, and environmental parameters of 113 barley accessions (Hordeum vulgare), previously selected based on high yield, in an irrigated production system in the Cerrado (Brazilian savanna), for use in breeding programs in Brazil. The experiment was conducted in 2013 at two sites in the Federal District, using a randomized complete block design with three replicates. The evaluated traits were: grain yield, kernel plumpness, thousand grain weight, plant height, lodging, and days to heading. Significant differences were observed between environment and accessions for all studied traits, as well as genotype x environment interaction. The MCU 3832 PI 402310, MCU 3484 PI 401962, CI 08053 Custer, MCU 3634 PI 402112, and MCU 3816 PI 402294 accessions stood out regarding grain yield and kernel plumpness. It is possible to obtain high selection gains with high heritability values. However, indirect selection is not recommended in the experimental conditions due to the low genotypic and phenotypic correlations obtained. It is also possible to select barley accessions with high yield and early heading date simultaneously, an important factor in the genotype selection process for future experiments. The accessions from Colombia are the most adapted to the Cerrado conditions.

Index terms: Hordeum vulgare, elite genotypes, genetic resources, malting quality, plant breeding.

\section{Caracterização agronômica de acessos de cevada de alta produtividade sob irrigação no Cerrado}

Resumo - O objetivo deste trabalho foi estimar parâmetros genéticos, fenotípicos e ambientais de 113 acessos de cevada (Hordeum vulgare), previamente selecionados com base em alta produtividade, em sistema de produção irrigado no Cerrado, para uso em programas de melhoramento no Brasil. O experimento foi realizado em 2013, em dois locais no Distrito Federal, tendo-se utilizado o delineamento experimental de blocos ao acaso, com três repetições. Avaliaram-se as características: rendimento de grãos, classificação comercial de primeira, peso de mil grãos, altura de plantas, acamamento e dias para espigamento. Foram observadas diferenças significativas entre ambientes e acessos para todas as características avaliadas, além de interação genótipo x ambiente. Os acessos MCU 3832 PI 402310, MCU 3484 PI 401962, CI 08053 Custer, MCU 3634 PI 402112 e MCU 3816 PI 402294 destacaram-se em relação ao rendimento de grãos e à classificação comercial de primeira. É possível obter altos ganhos de seleção com altos valores de herdabilidade. No entanto, a seleção indireta não é recomendada nas condições experimentais, em razão das baixas correlações genotípicas e fenotípicas obtidas. Também é possível a seleção simultânea de acessos de cevada com alto rendimento e ciclo de espigamento precoce, fator decisivo no processo de escolha de genótipos para futuros experimentos. Os acessos colombianos são os mais adaptados às condições de Cerrado.

Termos para indexação: Hordeum vulgare, genótipos de elite, recursos genéticos, qualidade de malteação, melhoramento de plantas.

\section{Introduction}

Barley is the fourth most grown cereal crop in the world, after corn, wheat, and rice. It is a plant with great adaptability, sown in several regions of the globe and at different latitudes. In Brazil, it is mainly sown in the South as a winter crop (Agostinetto et al., 2015). The country produces 317 thousand tons in 85 thousand hectares (Faostat, 2016), which only meets $43 \%$ of the demands of the Brazilian malt-production industry. For this reason, to supply the national beer industry, 350 thousand tons of barley are still imported 
yearly to reach the production of 1.3 million tons of malt (Ferreira et al., 2016).

Irrigated barley is cultivated in the states of Goiás, São Paulo, Minas Gerais, and in Distrito Federal, while dryland barley is grown in the states of the Southern region of Brazil, where the BRS Brau, BRS Elis, and BRS Cauê cultivars are used (Minella et al., 2013). Recently, other cultivars have been recommended for the Cerrado (Brazilian savanna) region, such as BRS 195, BRS Sampa, BRS - Deméter, BRS Manduri, and BRS Savanna (Amabile et al., 2014).

However, despite the availability of cultivars, Brazil still remains as one of the world's largest malt importers due to the small production in the country. In order to reduce this dependency, there is a continuous and growing demand for genotypes with malting quality adapted to the Cerrado region, which makes the selection of genotypes in this specific environment necessary (Amabile et al., 2013).

This shows that more knowledge about the genetic resources of barley is fundamental, especially when considering elite genotypes with good agronomic traits, originating from different plant breeding programs from several parts of the world. In this context, the characterization of genetic resources for breeding programs has been contributing significantly to the main qualitative and quantitative gains of Brazilian agriculture. More specifically, barley evaluation, selection, and breeding are important for the development of genetic materials that fill the requirements of the irrigated production system of the Cerrado, establishing the species as an economic and agronomic alternative for the region.

The objective of this work was to estimate the genetic, phenotypic, and environmental parameters of 113 barley accessions (Hordeum vulgare), previously selected based on high yield, in an irrigated production system in the Cerrado (Brazilian savanna), for use in breeding programs in Brazil.

\section{Materials and Methods}

A total of 113 hulled beer barley (Hordeum vulgare L.) acessions of two-row an six-row were evaluated, and the six-row cultivar BRS 180 was used as the control (Tables 1 and 2). Theses accessions were previously selected for grain yield in a study performed by Monteiro (2012) at Embrapa Cerrados, agronomically characterizing 435 accessions of barley from the germplasm bank of Embrapa Recursos Genéticos e Biotecnologia. The experiments were conducted from May to September 2013 in two sites in Distrito Federal, Brazil, under center pivot irrigation system. Site 1 is located in the experimental field of

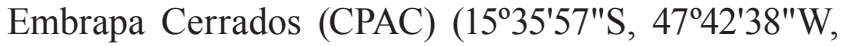
at an altitude of $1,007 \mathrm{~m}$ ), whereas site 2 is located at the experimental field of Embrapa Produtos e Mercado (SPM) $\left(15^{\circ} 54^{\prime} 53^{\prime \prime} \mathrm{S}, 48^{\circ} 02^{\prime} 14^{\prime \prime} \mathrm{W}\right.$, at an altitude of $1,254 \mathrm{~m})$. The soil of both sites is classified as a Latossolo Vermelho distrófico típico argiloso (Rhodic Hapludox). A randomized complete block design with three replicates was used. The plots consisted of six 5 -m long rows, spaced $20 \mathrm{~cm}$ apart, with a useful area of $4.8 \mathrm{~m}^{2}$ for each plot, with a density of 300 plants per square meter. According to the soil analysis, $16 \mathrm{~kg} \mathrm{ha}^{-1}$ $\mathrm{N}, 120 \mathrm{~kg} \mathrm{ha}^{-1} \mathrm{P}_{2} \mathrm{O}_{5}$, and $64 \mathrm{~kg} \mathrm{ha}^{-1} \mathrm{~K}_{2} \mathrm{O}$ were applied to the sowing groove, and $40 \mathrm{~kg} \mathrm{ha}^{-1} \mathrm{~N}$ were applied by the time the fifth fully expanded leaf arose (Amabile et al., 2007).

Six traits were evaluated: estimated grain yield $\left(\mathrm{kg} \mathrm{ha}^{-1}\right)$; kernel plumpness $(>2.5 \mathrm{~mm})$ in percentage, according to Brasil (1996); thousand grain weight (TSW) in grams (Regras..., 2009); plant height in centimeters, as the average of three plants per plot; degree of plant lodging (LOD), with data transformed into arc sine $X^{0.5} \times 100^{-1}$, in which $X$ is the value in percentage of lodging; and days to heading $(\mathrm{DH})$, which is the period, in days, from emergence to when $50 \%$ of the spikes inside the useful area of the plot were visible. The assessments of plant height, degrees of lodging, and heading were performed in the experimental fields of CPAC and SPM. The evaluations of grain yield, kernel plumpness, and TSW were carried out at the seed laboratory of Embrapa Cerrados.

The collected data were subjected to analysis of variance (Anova), and the averages were grouped by the Scott-Knott test, at $1 \%$ probability. Phenotypic variance $\left(\sigma_{\mathrm{f}}^{2}\right)$, genotypic quadratic component $\left(\sigma_{\mathrm{g}}^{2}\right)$, heritability $\left(\mathrm{h}^{2}\right)$, and the coefficients of environmental $(\mathrm{EVC})$, genetic $(\mathrm{GVC})$, and relative $(\mathrm{CVr})$ variance were estimated for each trait, using the Genes software (Universidade Federal de Viçosa, Viçosa, MG, Brazil). For the Anova, individual and group analyses were also performed for each trait.

The phenotypic, genotypic, and environmental correlations were measured based on the estimates of 
Table 1. Averages of 113 barley (Hordeum vulgare) accessions for different traits at the Embrapa Cerrados site.

\begin{tabular}{|c|c|c|c|c|c|c|c|c|c|c|}
\hline Genotype & Origin & $\begin{array}{c}\text { Yield } \\
\left(\mathrm{kg} \mathrm{ha}^{-1}\right)\end{array}$ & $\begin{array}{l}\text { PK } \\
(\%)\end{array}$ & $\begin{array}{l}\text { TSW } \\
(\mathrm{g})\end{array}$ & $\begin{array}{l}\text { Height } \\
(\mathrm{cm})\end{array}$ & $\begin{array}{c}\text { LOD } \\
(\operatorname{arc} \text { sine) }\end{array}$ & $\begin{array}{c}\mathrm{DH} \\
\text { (days) }\end{array}$ & Type & Cultivar & Color \\
\hline 2075C PI 371450 & Switzerland & $5,318.0 \mathrm{c}$ & $26.0 \mathrm{o}$ & $33.6 \mathrm{~g}$ & $79.0 \mathrm{~h}$ & $0.0 \mathrm{j}$ & $64.7 \mathrm{e}$ & 6 & Yes & Cream \\
\hline MCU 3592 PI 402070 & Colombia & $4,087.0 \mathrm{~g}$ & $51.3 \mathrm{i}$ & $40.0 \mathrm{e}$ & $86.0 \mathrm{e}$ & $0.0 \mathrm{j}$ & $52.7 \mathrm{~m}$ & 6 & No & Cream \\
\hline MCU 3458 PI 401936 & Colombia & $4,443.3 \mathrm{f}$ & 25.30 & $36.6 f$ & $64.3 n$ & $0.0977 \mathrm{a}$ & 54.01 & 6 & No & Cream \\
\hline MCU 3576 PI 402054 & Colombia & $4,520.3 \mathrm{e}$ & $12.0 \mathrm{q}$ & $31.6 \mathrm{~g}$ & $90.0 \mathrm{c}$ & $0.0 \mathrm{j}$ & 55.01 & 6 & Yes & Cream \\
\hline MCU 3832 PI 402310 & Colombia & $5,847.3 \mathrm{~b}$ & 42.61 & $33.3 \mathrm{~g}$ & $80.0 \mathrm{~g}$ & $0.0 \mathrm{j}$ & $60.0 \mathrm{~h}$ & 6 & No & Cream \\
\hline MCU 3452 PI 401930 & Colombia & $3,821.0 \mathrm{~g}$ & $61.6 \mathrm{~g}$ & $35.0 \mathrm{~g}$ & $64.0 \mathrm{n}$ & $0.0 \mathrm{j}$ & $56.3 \mathrm{k}$ & 6 & No & Cream \\
\hline H HOR 2325/58 PI 329126 & Afghanistan & $3,586.6 \mathrm{~h}$ & $35.6 \mathrm{~m}$ & 35.6 & 60.3 & $0.0 \mathrm{j}$ & $65.0 \mathrm{e}$ & 6 & Yes & Cream \\
\hline CI 15324 2260-85 & Tunisia & 3,391.6h & $58.6 \mathrm{~h}$ & $36.3 \mathrm{f}$ & 69.31 & $0.044 \mathrm{~h}$ & $65.0 \mathrm{e}$ & 6 & Yes & Cream \\
\hline MCU 3568 PI 402046 & Colombia & $5,330.6 \mathrm{c}$ & $44.3 \mathrm{k}$ & $33.0 \mathrm{~g}$ & 70.01 & $0.0733 \mathrm{~d}$ & $52.0 \mathrm{~m}$ & 6 & No & Cream \\
\hline E 3/416 PI 356495 & Ethiopia & $4,814.6 \mathrm{e}$ & $16.6 \mathrm{p}$ & $32.0 \mathrm{~g}$ & $57.6 \mathrm{p}$ & $0.1 \mathrm{a}$ & $61.7 \mathrm{~g}$ & 6 & Yes & Cream \\
\hline CI 15325 2244-87 & Tunisia & $4,215.0 \mathrm{f}$ & 40.61 & 37.0 & $78.3 \mathrm{~h}$ & $0.0363 \mathrm{~h}$ & $64.7 \mathrm{e}$ & 6 & Yes & Cream \\
\hline CI 06450 Oziery & Poland & $3,526.0 \mathrm{~h}$ & $33.0 \mathrm{n}$ & $28.3 \mathrm{~h}$ & $83.6 \mathrm{f}$ & $0.0 \mathrm{j}$ & $60.3 \mathrm{~h}$ & 6 & Yes & Cream \\
\hline CI 14857 ELS 6402-441 & Ethiopia & $1,905.3 \mathrm{j}$ & $51.0 \mathrm{f}$ & $36.6 \mathrm{f}$ & $78.0 \mathrm{~h}$ & $0.0 \mathrm{j}$ & $68.0 \mathrm{~b}$ & 6 & Yes & Cream \\
\hline MCU 3721 PI 402199 & Colombia & $2,845.6 \mathrm{i}$ & $51.6 \mathrm{~g}$ & $34.0 \mathrm{~g}$ & $76.6 \mathrm{i}$ & $0.0543 \mathrm{~g}$ & $63.7 \mathrm{f}$ & 6 & Yes & Cream \\
\hline MCU 3571 PI 402049 & Colombia & $4,735.0 \mathrm{e}$ & $61.0 \mathrm{~g}$ & $32.0 \mathrm{~g}$ & $75.6 \mathrm{i}$ & $0.0983 \mathrm{a}$ & $52.0 \mathrm{~m}$ & 6 & No & Cream \\
\hline CI 10018 Raspas Precoz 604 & Colombia & $3,484.0 \mathrm{~h}$ & $42.3 \mathrm{c}$ & $43.0 \mathrm{c}$ & $74.0 \mathrm{j}$ & $0.0977 \mathrm{a}$ & $56.3 \mathrm{k}$ & 6 & Yes & Cream \\
\hline CI 13069 Irba Moda & Ethiopia & $3,421.3 \mathrm{~h}$ & $11.6 \mathrm{q}$ & $36.0 \mathrm{f}$ & $88.0 \mathrm{~d}$ & $0.0977 \mathrm{a}$ & $57.3 \mathrm{j}$ & 6 & Yes & Cream \\
\hline CI 11684 & Afghanistan & $3,381.0 \mathrm{~h}$ & 29.30 & $38.6 \mathrm{e}$ & $77.3 \mathrm{i}$ & $0.0983 \mathrm{a}$ & $68.0 \mathrm{~b}$ & 6 & No & Cream \\
\hline CI 15296 Djebali 2316-57 & Tunisia & $2,759.6 \mathrm{i}$ & $46.3 \mathrm{k}$ & $41.6 \mathrm{~d}$ & $85.0 \mathrm{e}$ & $0.0977 \mathrm{a}$ & $66.0 \mathrm{~d}$ & 6 & Yes & Cream \\
\hline CI 07416 Dang - Bari 42 & South Korea & $1,536.3 \mathrm{j}$ & $53.3 \mathrm{i}$ & $33.0 \mathrm{~g}$ & $80.0 \mathrm{~g}$ & $0.044 \mathrm{~h}$ & $70.7 \mathrm{a}$ & 6 & Yes & Cream \\
\hline Galover (C A N 1126) PI 361636 & Denmark & $5,609.3 \mathrm{c}$ & $44.6 \mathrm{k}$ & $31.3 \mathrm{~g}$ & $82.3 \mathrm{f}$ & $0.0983 \mathrm{a}$ & $62.3 \mathrm{~g}$ & $2 / 6$ & Yes & Cream \\
\hline CI 10016 Raspa & Colombia & $3,942.3 \mathrm{~g}$ & $35.3 \mathrm{~m}$ & $32.3 \mathrm{~g}$ & $94.6 b$ & $0.0543 \mathrm{~g}$ & $65.3 \mathrm{e}$ & 6 & Yes & Cream \\
\hline MCU 3883 PI 402361 & Colombia & $4,318.0 \mathrm{f}$ & $31.6 n$ & $36.0 \mathrm{f}$ & $83.3 \mathrm{f}$ & $0.063 \mathrm{f}$ & 54.71 & 6 & Yes & Cream \\
\hline MCU 3858 PI 402336 & Colombia & $4,030.0 \mathrm{~g}$ & $71.0 \mathrm{e}$ & $40.3 d$ & $82.3 \mathrm{f}$ & $0.044 \mathrm{~h}$ & $58.7 \mathrm{i}$ & 6 & Yes & Cream \\
\hline MCU 3656 PI 402134 & Colombia & $4,374.0 \mathrm{f}$ & $32.3 n$ & $37.6 \mathrm{e}$ & $81.0 \mathrm{~g}$ & $0.0993 \mathrm{a}$ & $58.0 \mathrm{i}$ & 6 & No & Cream \\
\hline CI 14712 Orange Lemma & United States & $4,304.6 f$ & $26.0 \mathrm{o}$ & $27.6 \mathrm{~h}$ & $94.0 \mathrm{~b}$ & $0.0683 \mathrm{e}$ & $59.0 \mathrm{i}$ & $2 / 6$ & No & Cream \\
\hline MCU 3719 PI 402197 & Colombia & $4,235.0 \mathrm{f}$ & $64.6 \mathrm{f}$ & $40.3 \mathrm{~d}$ & $79.0 \mathrm{~h}$ & $0.0683 \mathrm{e}$ & $65.7 \mathrm{~d}$ & 6 & Yes & Cream \\
\hline CI 13683 Numar & United States & $5,959.3 b$ & $31.6 n$ & $44.6 \mathrm{c}$ & $84.0 \mathrm{f}$ & $0.0983 \mathrm{a}$ & $69.0 \mathrm{~b}$ & 6 & Yes & Cream \\
\hline CI 12920 & Ethiopia & $4,488.0 \mathrm{f}$ & $53.0 \mathrm{i}$ & $40.6 \mathrm{~d}$ & $85.3 \mathrm{e}$ & $0.0977 \mathrm{a}$ & $58.3 \mathrm{i}$ & 6 & Yes & Cream \\
\hline MCU 3653 PI 402131 & Colombia & $4,953.0 \mathrm{~d}$ & $61.0 \mathrm{~g}$ & $38.6 \mathrm{e}$ & $90.0 \mathrm{c}$ & $0.0983 a$ & 54.31 & 6 & Yes & Cream \\
\hline CI 06147 & Egypt & $3,866.6 \mathrm{~g}$ & $46.3 \mathrm{k}$ & $35.0 \mathrm{~g}$ & $97.3 \mathrm{a}$ & $0.0983 a$ & $67.0 \mathrm{c}$ & 6 & Yes & Cream \\
\hline CI 12916 & Ethiopia & $3,465.6 \mathrm{~h}$ & $52.6 \mathrm{i}$ & $39.3 \mathrm{e}$ & $94.0 \mathrm{~b}$ & $0.0993 \mathrm{a}$ & $65.7 \mathrm{~d}$ & 6 & Yes & Cream \\
\hline MCU 3780 PI 402258 & Colombia & $4,307.0 \mathrm{f}$ & $59.6 \mathrm{~g}$ & $36.6 f$ & $81.3 \mathrm{~g}$ & $0.0543 \mathrm{~g}$ & $64.7 \mathrm{e}$ & 6 & Yes & Cream \\
\hline CI 06188 & Mexico & $3,790.3 \mathrm{~g}$ & 41.01 & $35.6 f$ & $84.3 \mathrm{f}$ & $0.0993 \mathrm{a}$ & $67.3 \mathrm{c}$ & 6 & Yes & Cream \\
\hline MCU 3489 PI 401967 & Colombia & $4,134.0 \mathrm{f}$ & $68.0 \mathrm{f}$ & $40.3 d$ & $84.0 \mathrm{f}$ & $0.0683 \mathrm{e}$ & $57.0 \mathrm{j}$ & 6 & Yes & Cream \\
\hline CI 15560 QB 136-4-1 & Canada & $5,733.0 \mathrm{~b}$ & $49.0 \mathrm{j}$ & $33.0 \mathrm{~g}$ & $87.0 \mathrm{q}$ & $0.0983 a$ & $65.0 \mathrm{e}$ & 6 & No & Cream \\
\hline CI 09959 & Morocco & $4,952.6 \mathrm{~d}$ & $84.6 b$ & $45.0 \mathrm{c}$ & $73.0 \mathrm{q}$ & $0.0543 \mathrm{~g}$ & $57.3 \mathrm{j}$ & 6 & Yes & Cream \\
\hline CI $152802728-25$ & Tunisia & $3,071.3 \mathrm{i}$ & $46.0 \mathrm{k}$ & $38.0 \mathrm{r}$ & $81.0 \mathrm{~g}$ & $0.0993 \mathrm{a}$ & $67.3 \mathrm{c}$ & $2 / 6$ & Yes & Cream \\
\hline CI 10501 Athenais S-50-34 & Cyprus & $4,157.3 \mathrm{f}$ & $62.3 \mathrm{~g}$ & $35.6 \mathrm{f}$ & $79.6 \mathrm{~g}$ & $0.0977 \mathrm{a}$ & $58.0 \mathrm{i}$ & 6 & Yes & Cream \\
\hline CI 13894 & United States & $5,823.0 \mathrm{~b}$ & $51.0 \mathrm{i}$ & $36.0 \mathrm{i}$ & $76.0 \mathrm{i}$ & $0.0 \mathrm{j}$ & $60.3 \mathrm{~h}$ & 6 & No & Cream \\
\hline CI 12271 & Iran & $4,064.0 \mathrm{~g}$ & $45.3 \mathrm{k}$ & $29.3 \mathrm{j}$ & $73.6 j$ & $0.0483 \mathrm{~g}$ & $66.7 \mathrm{c}$ & 6 & Yes & Cream \\
\hline MCU 3876 PI 402354 & Colombia & $3,457.0 \mathrm{~h}$ & $32.6 n$ & $43.6 \mathrm{i}$ & $76.6 \mathrm{i}$ & $0.0683 \mathrm{e}$ & $52.7 \mathrm{~m}$ & 6 & Yes & Cream \\
\hline MCU 3827 PI 402305 & Colombia & $4,444.0 \mathrm{f}$ & $63.3 \mathrm{~g}$ & $41.0 \mathrm{~m}$ & $68.0 \mathrm{~m}$ & $0.088 \mathrm{~b}$ & $51.0 \mathrm{n}$ & 6 & No & Cream \\
\hline CI 09958 & Morocco & 3,199.0h & $87.6 b$ & $58.0 \mathrm{i}$ & $76.0 \mathrm{i}$ & $0.0617 f$ & $57.0 \mathrm{j}$ & 6 & Yes & Cream \\
\hline MCU 3469 PI 401947 & Colombia & $5,749.6 \mathrm{~b}$ & $75.0 \mathrm{~d}$ & $44.0 \mathrm{i}$ & $76.0 \mathrm{i}$ & $0.0407 \mathrm{~h}$ & $52.7 \mathrm{~m}$ & 6 & No & Cream \\
\hline MCU 3851 PI 402329 & Colombia & $5,828.6 \mathrm{~b}$ & $70.6 \mathrm{j}$ & $38.3 \mathrm{j}$ & $74.0 \mathrm{j}$ & $0.0993 \mathrm{a}$ & $63.7 \mathrm{f}$ & 6 & No & Cream \\
\hline MCU 3816 PI 402294 & Colombia & $5,067.6 \mathrm{~d}$ & $72.0 \mathrm{e}$ & $44.0 \mathrm{j}$ & $74.3 \mathrm{j}$ & $0.088 \mathrm{~b}$ & 54.31 & 6 & No & Cream \\
\hline CI 13715 & Colombia & $4,284.6 \mathrm{f}$ & $61.6 \mathrm{~g}$ & $44.3 \mathrm{c}$ & 61.60 & $0.0983 \mathrm{a}$ & $55.3 \mathrm{k}$ & 6 & No & Cream \\
\hline CI 10256 & Japan & $3,432.6 \mathrm{~h}$ & $67.0 \mathrm{f}$ & $46.0 \mathrm{c}$ & $63.3 n$ & $0.0983 a$ & $52.3 \mathrm{~m}$ & 6 & Yes & Cream \\
\hline CI 06946 & Iran & $5,234.6 \mathrm{~d}$ & $32.6 n$ & $34.0 \mathrm{~g}$ & $84.0 \mathrm{f}$ & $0.0977 \mathrm{a}$ & $60.7 \mathrm{~h}$ & 6 & Yes & Cream \\
\hline CI 07255 & United States & $5,245.6 \mathrm{~d}$ & $32.3 n$ & $34.0 \mathrm{~g}$ & $99.0 \mathrm{a}$ & $0.0983 \mathrm{a}$ & $60.0 \mathrm{~h}$ & 6 & No & Cream \\
\hline BRS 180 & Brazil & $6,142.0 \mathrm{a}$ & $73.6 \mathrm{~d}$ & $39.3 \mathrm{e}$ & $84.0 \mathrm{f}$ & $0.0 \mathrm{j}$ & $59.0 \mathrm{i}$ & 6 & Yes & Cream \\
\hline CI 15591 QB 139-1 & Canada & $4,736.0 \mathrm{e}$ & $62.3 \mathrm{~g}$ & 35.3 & $86.0 \mathrm{e}$ & $0.0977 \mathrm{a}$ & $57.7 \mathrm{j}$ & 6 & No & Cream \\
\hline CI 07607 & India & $4,148.3 \mathrm{f}$ & $38.0 \mathrm{~m}$ & $39.0 \mathrm{e}$ & 69.01 & $0.0977 \mathrm{a}$ & $55.3 \mathrm{k}$ & 6 & No & Cream \\
\hline MCU 3556 PI 402034 & Colombia & $3,455.3 \mathrm{~h}$ & $71.6 \mathrm{e}$ & $42.0 \mathrm{~d}$ & $87.6 \mathrm{~d}$ & $0.0 \mathrm{j}$ & $64.0 \mathrm{f}$ & 6 & No & Cream \\
\hline CI 07156 & United States & $3,830.3 \mathrm{~g}$ & $36.3 \mathrm{~m}$ & $38.6 \mathrm{e}$ & $76.0 \mathrm{i}$ & $0.0993 \mathrm{a}$ & $69.3 b$ & 6 & No & Cream \\
\hline CI 10078 Atlas 57 & United States & $5,516.6 \mathrm{c}$ & $85.0 \mathrm{~b}$ & $43.0 \mathrm{c}$ & $85.6 \mathrm{e}$ & $0.0543 \mathrm{~g}$ & $68.7 \mathrm{~b}$ & 6 & Yes & Cream \\
\hline
\end{tabular}

Continuation... 
Continuation...

\begin{tabular}{|c|c|c|c|c|c|c|c|c|c|c|}
\hline Genotype & Origin & $\begin{array}{c}\text { Yield } \\
\left(\mathrm{kg} \mathrm{ha}^{-1}\right)\end{array}$ & $\begin{array}{l}\text { PK } \\
(\%)\end{array}$ & $\begin{array}{l}\text { TSW } \\
(\mathrm{g})\end{array}$ & $\begin{array}{l}\text { Height } \\
(\mathrm{cm})\end{array}$ & $\begin{array}{c}\text { LOD } \\
\text { (arc sine) }\end{array}$ & $\begin{array}{c}\mathrm{DH} \\
\text { (days) }\end{array}$ & Type & Cultivar & Color \\
\hline CI 11493 Frugherste Stankas & Germany & $4,728.0 \mathrm{e}$ & $82.0 \mathrm{c}$ & $49.0 \mathrm{~b}$ & $67.0 \mathrm{~m}$ & $0.0 \mathrm{j}$ & $66.0 \mathrm{~d}$ & 2 & Yes & Cream \\
\hline CI 09882 Gondar & Ethiopia & $4,787.0 \mathrm{e}$ & $35.0 \mathrm{~m}$ & $44.3 \mathrm{c}$ & $82.0 \mathrm{f}$ & $0.0407 \mathrm{~h}$ & $70.7 \mathrm{a}$ & 6 & Yes & Cream \\
\hline CI 12598 & Ethiopia & $3,230.6 \mathrm{~h}$ & $49.3 \mathrm{j}$ & $48.0 \mathrm{~b}$ & 70.31 & $0.056 \mathrm{~g}$ & $55.7 \mathrm{k}$ & 6 & Yes & Cream \\
\hline CI 10082 Weibull S 4468 & Sweden & $3,496.6 \mathrm{~h}$ & $56.3 \mathrm{~h}$ & $41.3 \mathrm{~d}$ & $79.0 \mathrm{~h}$ & $0.0977 \mathrm{a}$ & $67.3 \mathrm{c}$ & 2 & Yes & Cream \\
\hline CI 15565 QB 136-20 & Canada & $4,861.3 \mathrm{e}$ & $71.6 \mathrm{e}$ & $42.3 \mathrm{~d}$ & $84.0 \mathrm{f}$ & $0.0943 \mathrm{a}$ & $67.0 \mathrm{c}$ & 6 & No & Cream \\
\hline CI 14925 ELS 6402-512 & Ethiopia & $4,171.6 f$ & $56.3 \mathrm{~h}$ & $44.6 \mathrm{c}$ & $79.0 \mathrm{~h}$ & $0.0993 \mathrm{a}$ & $66.0 \mathrm{~d}$ & 6 & Yes & Cream \\
\hline 2043C PI 371415 & Switzerland & $2,823.3 \mathrm{i}$ & $53.3 \mathrm{i}$ & $41.0 \mathrm{~d}$ & $67.0 \mathrm{~m}$ & $0.0967 \mathrm{a}$ & $66.0 \mathrm{~d}$ & 6 & Yes & Cream \\
\hline CI 09961 & Iran & $5,553.3 \mathrm{c}$ & $85.3 b$ & $48.6 \mathrm{~b}$ & $80.0 \mathrm{~g}$ & $0.088 \mathrm{~b}$ & $60.3 \mathrm{~h}$ & 6 & Yes & Cream \\
\hline CI 08053 Custer & United States & $5,719.3 b$ & $56.0 \mathrm{~h}$ & $43.3 \mathrm{c}$ & $88.6 \mathrm{~d}$ & $0.0993 \mathrm{a}$ & $67.3 \mathrm{c}$ & 6 & Yes & Cream \\
\hline MCU 3461 PI 401939 & Colombia & $3,644.3 \mathrm{~h}$ & $68.0 \mathrm{f}$ & $43.3 \mathrm{c}$ & $91.0 \mathrm{c}$ & $0.088 \mathrm{~b}$ & $62.0 \mathrm{~g}$ & 6 & No & Cream \\
\hline MCU 3484 PI 401962 & Colombia & $6,508.3 \mathrm{a}$ & $71.6 \mathrm{e}$ & $34.3 \mathrm{~g}$ & $86.0 \mathrm{e}$ & $0.0787 \mathrm{~d}$ & $50.3 n$ & 6 & No & Cream \\
\hline CI 14031 & Ethiopia & $3,719.0 \mathrm{~g}$ & $56.6 \mathrm{~h}$ & $40.0 \mathrm{e}$ & $83.6 \mathrm{f}$ & $0.0603 \mathrm{f}$ & $67.0 \mathrm{c}$ & 6 & Yes & Cream \\
\hline CI 10017 Raspa Comun 1085 & Colombia & $4,193.6 f$ & $70.0 \mathrm{e}$ & $43.6 \mathrm{c}$ & $78.0 \mathrm{~h}$ & $0.0983 \mathrm{a}$ & $65.7 \mathrm{~d}$ & 6 & Yes & Cream \\
\hline CI $152792528-23$ & Tunisia & $5,430.0 \mathrm{c}$ & $72.6 \mathrm{~d}$ & $45.6 \mathrm{c}$ & $83.0 \mathrm{f}$ & $0.0977 \mathrm{a}$ & $65.7 \mathrm{~d}$ & 6 & Yes & Cream \\
\hline CI 15302 1356-33 & Tunisia & $3,550.0 \mathrm{~h}$ & 40.31 & $38.3 \mathrm{e}$ & $85.0 \mathrm{e}$ & $0.0993 \mathrm{a}$ & $63.7 f$ & 6 & Yes & Cream \\
\hline MCU 3454 PI 401932 & Colombia & $4,600.0 \mathrm{e}$ & $71.3 \mathrm{e}$ & $44.3 \mathrm{c}$ & $85.0 \mathrm{e}$ & $0.0933 a$ & $60.7 \mathrm{~h}$ & 6 & No & Cream \\
\hline CI 15580 QB $136-41$ & Canada & $5,983.0 \mathrm{~b}$ & $73.3 \mathrm{~d}$ & $45.3 \mathrm{c}$ & $95.0 \mathrm{~b}$ & $0.0757 \mathrm{~d}$ & $62.7 \mathrm{~g}$ & 6 & No & Cream \\
\hline CI 07772 & India & $5,005.0 \mathrm{~d}$ & $61.0 \mathrm{~g}$ & $41.3 \mathrm{~d}$ & 69.01 & $0.088 \mathrm{~b}$ & $52.0 \mathrm{~m}$ & 6 & Yes & Cream \\
\hline CI 14041 & Ethiopia & $3,629.3 \mathrm{~h}$ & $68.6 \mathrm{f}$ & $46.0 \mathrm{c}$ & $80.0 \mathrm{~g}$ & $0.0517 \mathrm{~g}$ & $68.7 \mathrm{~b}$ & 6 & Yes & Cream \\
\hline MCU 3467 PI 401945 & Colombia & $4,593.3 \mathrm{e}$ & $71.6 \mathrm{e}$ & $40.3 \mathrm{~d}$ & $87.6 \mathrm{~d}$ & $0.0603 \mathrm{f}$ & $62.7 \mathrm{~g}$ & 6 & No & Cream \\
\hline CI 06109 Velvon & United States & $4,987.3 \mathrm{~d}$ & $70.0 \mathrm{e}$ & $40.6 \mathrm{~d}$ & $79.3 \mathrm{~h}$ & $0.0763 \mathrm{~d}$ & $68.7 b$ & 6 & Yes & Cream \\
\hline MCU 3478 PI 401956 & Colombia & $5,524.6 \mathrm{c}$ & $71.6 \mathrm{e}$ & $36.0 \mathrm{f}$ & $83.0 \mathrm{f}$ & $0.085 \mathrm{~b}$ & $60.0 \mathrm{~h}$ & 6 & No & Cream \\
\hline CI 09962 & Iran & $4,671.0 \mathrm{e}$ & $91.6 \mathrm{a}$ & $57.0 \mathrm{a}$ & 69.31 & $0.063 \mathrm{f}$ & $60.3 \mathrm{~h}$ & 6 & Yes & Cream \\
\hline MCU 3878 PI 402356 & Colombia & $4,234.6 f$ & $60.0 \mathrm{~g}$ & $39.6 \mathrm{e}$ & $73.0 \mathrm{j}$ & $0.0603 \mathrm{f}$ & $53.3 \mathrm{~m}$ & 6 & No & Cream \\
\hline CI 10697 & Iran & $4,642.6 \mathrm{e}$ & 42.61 & $48.3 b$ & $79.6 \mathrm{~g}$ & $0.0977 \mathrm{a}$ & $57.0 \mathrm{j}$ & 6 & Yes & Cream \\
\hline CI 15323 2222-79 & Tunisia & $3,636.0 \mathrm{~h}$ & $70.0 \mathrm{e}$ & $48.6 b$ & $79.6 \mathrm{~g}$ & $0.0983 \mathrm{a}$ & $65.7 \mathrm{~d}$ & 6 & Yes & Cream \\
\hline MCU 3750 PI 402228 & Colombia & $4,634.0 \mathrm{e}$ & $84.3 b$ & $47.6 \mathrm{~b}$ & $72.0 \mathrm{k}$ & $0.0423 \mathrm{~h}$ & $63.7 \mathrm{f}$ & 6 & No & Cream \\
\hline CI 12918 & Ethiopia & $4,471.3 \mathrm{f}$ & $82.3 \mathrm{c}$ & $48.6 \mathrm{~b}$ & $76.0 \mathrm{i}$ & $0.0977 \mathrm{a}$ & $62.7 \mathrm{~g}$ & 6 & Yes & Cream \\
\hline MCU 3634 PI 402112 & Colombia & $6,031.6 \mathrm{~b}$ & $70.3 \mathrm{e}$ & $35.6 \mathrm{f}$ & $83.3 \mathrm{f}$ & $0.0977 \mathrm{a}$ & 54.71 & 6 & No & Cream \\
\hline E 273/4 PI 356414 & Ethiopia & $3,845.3 \mathrm{~g}$ & $61.6 \mathrm{~g}$ & $45.0 \mathrm{c}$ & 59.60 & $0.063 \mathrm{f}$ & $60.7 \mathrm{~h}$ & 6 & Yes & Cream \\
\hline Carina PI 371632 & Germany & $4,050.0 \mathrm{~g}$ & $84.0 \mathrm{~b}$ & $40.6 \mathrm{~d}$ & $80.0 \mathrm{~g}$ & $0.0 \mathrm{j}$ & $67.7 \mathrm{c}$ & 2 & Yes & Cream \\
\hline CI 15281 3102-16 & Tunisia & $4,116.6 f$ & $52.6 \mathrm{i}$ & $41.0 \mathrm{~d}$ & $84.6 \mathrm{e}$ & $0.0993 \mathrm{a}$ & $67.7 \mathrm{c}$ & $2 / 6$ & Yes & Cream \\
\hline CI 02109 White Smyrna & United States & $4,230.3 \mathrm{f}$ & $68.3 \mathrm{f}$ & $46.6 b$ & $85.6 \mathrm{e}$ & $0.0983 \mathrm{a}$ & $60.7 \mathrm{~h}$ & 2 & Yes & Cream \\
\hline MCU 3865 PI 402343 & Colombia & $4,939.6 \mathrm{~d}$ & $73.0 \mathrm{~d}$ & $45.3 \mathrm{c}$ & $74.0 \mathrm{j}$ & $0.0407 \mathrm{~h}$ & $61.3 \mathrm{~h}$ & 6 & No & Cream \\
\hline CI 13704 & England & $3,309.0 \mathrm{~h}$ & $70.0 \mathrm{e}$ & $48.6 b$ & 83.0f & $0.0577 \mathrm{~g}$ & $58.0 \mathrm{i}$ & 2 & Yes & Black \\
\hline CI 12367 Branisovicky & Czech Republic & $5,275.3 \mathrm{~d}$ & $81.0 \mathrm{c}$ & $40.0 \mathrm{e}$ & $76.0 \mathrm{i}$ & $0.02 \mathrm{i}$ & $67.3 \mathrm{c}$ & 2 & Yes & Cream \\
\hline CI 12262 & Iran & $3,988.0 \mathrm{~g}$ & $52.3 \mathrm{i}$ & $44.0 \mathrm{c}$ & $78.6 \mathrm{~h}$ & $0.084 \mathrm{c}$ & $64.3 \mathrm{e}$ & 6 & Yes & Cream \\
\hline CI 10114 Carlsberg II & Denmark & $2,943.0 \mathrm{i}$ & $58.3 \mathrm{~h}$ & $42.3 \mathrm{~d}$ & $73.0 \mathrm{j}$ & $0.0603 \mathrm{f}$ & $66.3 d$ & 2 & Yes & Cream \\
\hline MCU 3852 PI 402330 & Colombia & $4,541.0 \mathrm{e}$ & $74.0 \mathrm{~d}$ & $39.6 \mathrm{e}$ & 69.61 & $0.0387 \mathrm{~h}$ & $60.3 \mathrm{~h}$ & 6 & No & Cream \\
\hline A HOR 55/61 PI 327910 & Bulgaria & $4,262.3 \mathrm{f}$ & $48.3 \mathrm{j}$ & $39.6 \mathrm{e}$ & $72.6 \mathrm{j}$ & $0.088 \mathrm{~b}$ & $60.7 \mathrm{~h}$ & 6 & Yes & Cream \\
\hline CI 10140 & Afghanistan & $4,642.6 \mathrm{e}$ & $72.3 \mathrm{e}$ & $40.6 \mathrm{~d}$ & $77.6 \mathrm{~h}$ & $0.0533 \mathrm{~g}$ & 54.31 & 6 & Yes & Cream \\
\hline MCU 3884 PI 402362 & Colombia & $5,111.0 \mathrm{~d}$ & $61.0 \mathrm{~g}$ & $44.6 \mathrm{c}$ & $64.3 n$ & $0.0983 \mathrm{a}$ & $52.3 \mathrm{~m}$ & 6 & No & Cream \\
\hline MCU 3778 PI 402256 & Colombia & $4,656.6 \mathrm{e}$ & $71.6 \mathrm{e}$ & $39.0 \mathrm{e}$ & $94.0 \mathrm{~b}$ & $0.096 \mathrm{a}$ & $63.0 \mathrm{~g}$ & 6 & Yes & Cream \\
\hline CI 09952 & Russia & $4,322.0 \mathrm{f}$ & $82.0 \mathrm{c}$ & $44.6 \mathrm{c}$ & $84.0 \mathrm{f}$ & $0.088 \mathrm{~b}$ & $57.7 \mathrm{j}$ & 6 & Yes & Cream \\
\hline MCU 3448 PI 401926 & Colombia & $5,635.3 \mathrm{c}$ & $59.3 \mathrm{~g}$ & $38.6 \mathrm{e}$ & $88.6 \mathrm{~d}$ & $0.0363 \mathrm{~h}$ & $55.7 \mathrm{k}$ & 6 & No & Cream \\
\hline CI 06244 & United States & $5,136.3 d$ & $71.0 \mathrm{e}$ & $40.6 \mathrm{~d}$ & $76.0 \mathrm{i}$ & $0.0983 \mathrm{a}$ & $60.3 \mathrm{~h}$ & 6 & Yes & Cream \\
\hline MCU 3449 PI 401927 & Colombia & $5,767.6 \mathrm{~b}$ & $72.3 \mathrm{e}$ & $50.0 \mathrm{~b}$ & $82.3 \mathrm{f}$ & $0.0 \mathrm{j}$ & $61.7 \mathrm{~g}$ & 6 & No & Cream \\
\hline MCU 3654 PI 402132 & Colombia & $5,065.6 \mathrm{~d}$ & $79.6 \mathrm{c}$ & $38.3 \mathrm{e}$ & $79.0 \mathrm{~h}$ & $0.088 \mathrm{~b}$ & 54.31 & 6 & No & Cream \\
\hline CI 12068 Mazowiecki & Poland & $5,128.3 \mathrm{~d}$ & $68.6 \mathrm{f}$ & $33.3 \mathrm{~g}$ & $81.3 \mathrm{~g}$ & $0.096 \mathrm{a}$ & $60.7 \mathrm{~h}$ & 6 & Yes & Cream \\
\hline MCU 3502 PI 401980 & Colombia & $5,147.3 \mathrm{~d}$ & $81.0 \mathrm{c}$ & $43.3 \mathrm{c}$ & $87.0 \mathrm{~d}$ & $0.0917 \mathrm{~b}$ & $58.7 \mathrm{i}$ & 6 & No & Cream \\
\hline MCU 3870 PI 402348 & Colombia & $5,237.6 \mathrm{~d}$ & $74.3 d$ & $38.3 \mathrm{e}$ & $94.3 b$ & $0.0993 \mathrm{a}$ & $60.3 \mathrm{~h}$ & 6 & No & Cream \\
\hline CI 10071 Wolfe & Canada & $5,337.6 \mathrm{c}$ & $74.3 \mathrm{~d}$ & $39.0 \mathrm{e}$ & $91.6 \mathrm{c}$ & $0.0977 \mathrm{a}$ & $60.0 \mathrm{~h}$ & 6 & Yes & Cream \\
\hline CI 13711 & Colombia & $5,533.6 \mathrm{c}$ & $72.0 \mathrm{e}$ & $41.0 \mathrm{~d}$ & $84.0 \mathrm{f}$ & $0.0993 \mathrm{a}$ & $59.7 \mathrm{~h}$ & 6 & No & Cream \\
\hline CI 10022 & Colombia & $5,444.0 \mathrm{c}$ & $69.3 \mathrm{e}$ & $42.3 \mathrm{~d}$ & 69.61 & $0.0977 \mathrm{a}$ & $58.7 \mathrm{i}$ & 6 & Yes & Cream \\
\hline CI 13824 Atlas 68 & United States & $4,314.3 \mathrm{f}$ & $75.0 \mathrm{~d}$ & $44.3 c$ & $79.0 \mathrm{~h}$ & $0.095 \mathrm{a}$ & $63.3 \mathrm{~g}$ & 6 & Yes & Cream \\
\hline MCU 3850 PI 402328 & Colombia & $5,446.6 \mathrm{c}$ & $85.6 \mathrm{~b}$ & $44.6 \mathrm{c}$ & $78.6 \mathrm{~h}$ & $0.089 \mathrm{~b}$ & $61.0 \mathrm{~h}$ & 6 & Yes & Cream \\
\hline
\end{tabular}

${ }^{(1)}$ Yield, grain yield, PK; kernel plumpness; TSW, thousand seed weight; Height, plant height; LOD, lodging; and DH, days to heading. Different letters next to treatments indicate significant differences by the Scott-Knott test, at $1 \%$ probability. 
Table 2. Averages of 113 barley (Hordeum vulgare) accessions for different traits at the Embrapa Produtos e Mercado site.

\begin{tabular}{|c|c|c|c|c|c|c|c|c|c|c|}
\hline Genotype & Origin & $\begin{array}{c}\text { Yield } \\
\left(\mathrm{kg} \mathrm{ha}^{-1}\right)\end{array}$ & $\begin{array}{l}\text { PK } \\
(\%)\end{array}$ & $\begin{array}{c}\text { TSW } \\
(\mathrm{g})\end{array}$ & $\begin{array}{l}\text { Height } \\
(\mathrm{cm})\end{array}$ & $\begin{array}{c}\text { LOD } \\
(\operatorname{arcsine})\end{array}$ & $\begin{array}{c}\mathrm{DH} \\
\text { (days) }\end{array}$ & Type & Cultivar & Color \\
\hline 2075 C PI 371450 & Switzerland & $6,009 \mathrm{i}$ & $70.6 \mathrm{c}$ & $46.0 \mathrm{e}$ & $85.0 \mathrm{c}$ & $0.0107 \mathrm{e}$ & $51.0 \mathrm{i}$ & 6 & Yes & Cream \\
\hline MCU 3592 PI 402070 & Colombia & $6,468 \mathrm{~g}$ & $77.3 \mathrm{~b}$ & $33.3 \mathrm{~h}$ & $94.3 \mathrm{a}$ & $0.0000 \mathrm{f}$ & $50.3 \mathrm{j}$ & 6 & No & Cream \\
\hline MCU 3458 PI 401936 & Colombia & $5,530 \mathrm{k}$ & $53.0 \mathrm{e}$ & $48.0 \mathrm{~d}$ & $74.0 \mathrm{e}$ & $0.1000 \mathrm{a}$ & $50.0 \mathrm{j}$ & 6 & No & Cream \\
\hline MCU 3576 PI 402054 & Colombia & 5,0611 & $52.3 \mathrm{e}$ & $46.0 \mathrm{e}$ & $96.3 \mathrm{a}$ & $0.0107 \mathrm{e}$ & $49.6 \mathrm{j}$ & 6 & Yes & Cream \\
\hline MCU 3832 PI 402310 & Colombia & $7,695 b$ & $78.6 \mathrm{~b}$ & $41.0 \mathrm{f}$ & $87.6 \mathrm{~b}$ & $0.0000 \mathrm{f}$ & $50.6 \mathrm{j}$ & 6 & No & Cream \\
\hline MCU 3452 PI 401930 & Colombia & $7,154 d$ & $88.3 \mathrm{a}$ & $51.0 \mathrm{c}$ & $77.6 \mathrm{~d}$ & $0.0000 \mathrm{f}$ & $51.0 \mathrm{i}$ & 6 & No & Cream \\
\hline H HOR 2325/58 PI 329126 & Afghanistan & $9,187 \mathrm{a}$ & $78.3 \mathrm{~b}$ & $43.6 \mathrm{f}$ & $74.3 \mathrm{e}$ & $0.0000 \mathrm{f}$ & $53.0 \mathrm{~h}$ & 6 & Yes & Cream \\
\hline CI $153242260-85$ & Tunisia & $5,731 \mathrm{j}$ & $71.0 \mathrm{c}$ & $46.3 \mathrm{e}$ & $77.3 d$ & $0.0720 \mathrm{~b}$ & $52.0 \mathrm{i}$ & 6 & Yes & Cream \\
\hline MCU 3568 PI 402046 & Colombia & $7,241 \mathrm{~d}$ & $60.3 \mathrm{~d}$ & $42.3 \mathrm{f}$ & $83.0 \mathrm{c}$ & $0.1000 \mathrm{a}$ & $49.6 \mathrm{j}$ & 6 & No & Cream \\
\hline E 3/416 PI 356495 & Ethiopia & $7,082 \mathrm{e}$ & $79.0 \mathrm{~b}$ & $39.3 \mathrm{~g}$ & $69.0 \mathrm{e}$ & $0.1000 \mathrm{a}$ & $52.6 \mathrm{~h}$ & 6 & Yes & Cream \\
\hline CI 15325 2244-87 & Tunisia & $4,784 \mathrm{~m}$ & $70.3 \mathrm{c}$ & $47.3 \mathrm{e}$ & $85.0 \mathrm{c}$ & $0.0500 \mathrm{~d}$ & $50.0 \mathrm{j}$ & 6 & Yes & Cream \\
\hline CI 06450 Oziery & Poland & $6,272 \mathrm{~h}$ & $67.0 \mathrm{c}$ & $39.3 \mathrm{~g}$ & $87.6 \mathrm{~b}$ & $0.0107 \mathrm{e}$ & $50.6 \mathrm{j}$ & 6 & Yes & Cream \\
\hline CI 14857 ELS 6402-441 & Ethiopia & 5,3481 & $63.6 \mathrm{c}$ & $43.3 \mathrm{f}$ & $88.0 \mathrm{~b}$ & $0.0107 \mathrm{e}$ & $56.6 \mathrm{f}$ & 6 & Yes & Cream \\
\hline MCU 3721 PI 402199 & Colombia & $5,583 \mathrm{k}$ & $90.3 \mathrm{a}$ & $53.0 \mathrm{c}$ & $86.0 \mathrm{c}$ & $0.0670 \mathrm{c}$ & $56.0 \mathrm{~g}$ & 6 & Yes & Cream \\
\hline MCU 3571 PI 402049 & Colombia & $6,533 \mathrm{~g}$ & $86.6 \mathrm{a}$ & $44.3 \mathrm{f}$ & $85.0 \mathrm{c}$ & $0.1000 \mathrm{a}$ & $49.3 \mathrm{j}$ & 6 & No & Cream \\
\hline CI 10018 Raspa Precoz 604 & Colombia & $6,669 f$ & $72.0 \mathrm{c}$ & $44.6 \mathrm{e}$ & $84.3 \mathrm{c}$ & $0.1000 \mathrm{a}$ & $52.0 \mathrm{i}$ & 6 & Yes & Cream \\
\hline CI 13069 Irba Moda & Ethiopia & 5,3041 & $40.6 \mathrm{e}$ & $48.0 \mathrm{~d}$ & $91.0 \mathrm{~b}$ & $0.1000 \mathrm{a}$ & $51.6 \mathrm{i}$ & 6 & Yes & Cream \\
\hline CI 11684 & Afghanistan & 5,2191 & $67.6 \mathrm{c}$ & $43.0 \mathrm{f}$ & $87.0 \mathrm{~b}$ & $0.1000 \mathrm{a}$ & $58.0 \mathrm{e}$ & 6 & No & Cream \\
\hline CI 15296 Djebali 2316-57 & Tunisia & 5,3811 & $83.6 \mathrm{a}$ & $54.0 \mathrm{c}$ & $90.0 \mathrm{~b}$ & $0.1000 \mathrm{a}$ & $58.0 \mathrm{e}$ & 6 & Yes & Cream \\
\hline CI 07416 Dang - Bari 42 & South Korea & 3,9230 & $77.3 \mathrm{~b}$ & $32.3 \mathrm{~h}$ & $87.3 \mathrm{~b}$ & $0.0630 \mathrm{c}$ & $58.3 \mathrm{e}$ & 6 & Yes & Cream \\
\hline GALOVER (C A N 1126) PI 361636 & Denmark & $5,815 \mathrm{j}$ & $81.0 \mathrm{~b}$ & $36.6 \mathrm{~g}$ & $90.0 \mathrm{~b}$ & $0.1000 \mathrm{a}$ & $51.0 \mathrm{i}$ & $2 / 6$ & Yes & Cream \\
\hline CI 10016 Raspa & Colombia & $4,761 \mathrm{~m}$ & $84.6 \mathrm{a}$ & $52.6 \mathrm{c}$ & $98.3 \mathrm{a}$ & $0.0707 \mathrm{~b}$ & $53.3 \mathrm{~h}$ & 6 & Yes & Cream \\
\hline MCU 3883 PI 402361 & Colombia & $6,138 \mathrm{~h}$ & $69.3 \mathrm{c}$ & $46.3 \mathrm{e}$ & $92.3 b$ & $0.0870 \mathrm{a}$ & $54.3 \mathrm{~h}$ & 6 & Yes & Cream \\
\hline MCU 3858 PI 402336 & Colombia & $6,078 \mathrm{i}$ & $82.6 \mathrm{~b}$ & $45.3 \mathrm{e}$ & $87.6 \mathrm{~b}$ & $0.0543 \mathrm{c}$ & $54.0 \mathrm{~h}$ & 6 & Yes & Cream \\
\hline MCU 3656 PI 402134 & Colombia & 5,0251 & $47.0 \mathrm{e}$ & $42.0 \mathrm{f}$ & $92.3 b$ & $0.1000 \mathrm{a}$ & $54.3 \mathrm{~h}$ & 6 & No & Cream \\
\hline CI 14712 Orange Lemma & United States & $4,801 \mathrm{~m}$ & $48.3 \mathrm{e}$ & $42.6 \mathrm{f}$ & $97.6 \mathrm{a}$ & $0.0830 \mathrm{~b}$ & $53.3 \mathrm{~h}$ & $2 / 6$ & No & purple \\
\hline MCU 3719 PI 402197 & Colombia & $6,433 \mathrm{~g}$ & $88.6 \mathrm{a}$ & $52.6 \mathrm{c}$ & $87.6 \mathrm{~b}$ & $0.0880 \mathrm{a}$ & $55.0 \mathrm{~g}$ & 6 & Yes & Cream \\
\hline CI 13683 Numar & United States & $7,049 \mathrm{e}$ & $76.0 \mathrm{~b}$ & $45.6 \mathrm{e}$ & $89.3 b$ & $0.1000 \mathrm{a}$ & $58.0 \mathrm{e}$ & 6 & Yes & Cream \\
\hline CI 12920 & Ethiopia & $6,268 \mathrm{~h}$ & $76.6 \mathrm{~b}$ & $53.6 \mathrm{c}$ & $90.0 \mathrm{~b}$ & $0.1000 \mathrm{a}$ & $61.6 \mathrm{c}$ & 6 & Yes & Cream \\
\hline MCU 3653 PI 402131 & Colombia & $6,533 \mathrm{~g}$ & $74.0 \mathrm{~b}$ & $45.6 \mathrm{e}$ & $96.3 \mathrm{a}$ & $0.1000 \mathrm{a}$ & $62.3 \mathrm{c}$ & 6 & Yes & Cream \\
\hline CI 06147 & Egypt & $4,110 \mathrm{o}$ & $58.6 \mathrm{~d}$ & $47.0 \mathrm{e}$ & $98.0 \mathrm{a}$ & $0.1000 \mathrm{a}$ & $63.6 b$ & 6 & Yes & Cream \\
\hline CI 12916 & Ethiopia & $6,256 \mathrm{~h}$ & $68.0 \mathrm{c}$ & $46.6 \mathrm{e}$ & $96.0 \mathrm{a}$ & $0.1000 \mathrm{a}$ & $62.0 \mathrm{c}$ & 6 & Yes & Cream \\
\hline MCU 3780 PI 402258 & Colombia & $6,258 \mathrm{~h}$ & $68.0 \mathrm{c}$ & $46.3 \mathrm{e}$ & $87.6 \mathrm{~b}$ & $0.0777 \mathrm{~b}$ & $60.6 \mathrm{~d}$ & 6 & Yes & Cream \\
\hline CI 06188 & Mexico & $6,234 \mathrm{~h}$ & $80.6 \mathrm{~b}$ & $45.3 \mathrm{e}$ & $91.3 b$ & $0.1000 \mathrm{a}$ & $61.0 \mathrm{~d}$ & 6 & Yes & Cream \\
\hline MCU 3489 PI 401967 & Colombia & $6,140 \mathrm{~h}$ & $87.6 \mathrm{a}$ & $46.3 \mathrm{e}$ & $87.3 b$ & $0.1000 \mathrm{a}$ & $55.6 \mathrm{~g}$ & 6 & Yes & Cream \\
\hline CI 15560 QB 136-4-1 & Canada & $6,124 \mathrm{~h}$ & $80.0 \mathrm{~b}$ & $51.3 \mathrm{c}$ & $84.0 \mathrm{c}$ & $0.1000 \mathrm{a}$ & $51.0 \mathrm{i}$ & 6 & No & Cream \\
\hline CI 09959 & Morocco & $6,133 \mathrm{~h}$ & $85.3 \mathrm{a}$ & $51.0 \mathrm{c}$ & $85.3 \mathrm{c}$ & $0.0630 \mathrm{c}$ & $61.0 \mathrm{~d}$ & 6 & Yes & Cream \\
\hline CI $152802728-25$ & Tunisia & 5,1331 & $76.3 b$ & $46.0 \mathrm{e}$ & $85.3 \mathrm{c}$ & $0.1000 \mathrm{a}$ & $63.6 b$ & $2 / 6$ & Yes & Cream \\
\hline CI 10501 Athenais S-50-34 & Cyprus & $5,695 \mathrm{j}$ & $89.3 \mathrm{a}$ & $54.3 \mathrm{c}$ & $88.3 b$ & $0.1000 \mathrm{a}$ & $56.6 f$ & 6 & Yes & Cream \\
\hline CI 13894 & United States & $6,191 \mathrm{~h}$ & $76.6 \mathrm{~b}$ & $37.3 \mathrm{~g}$ & $82.0 \mathrm{c}$ & $0.0000 \mathrm{f}$ & $57.6 \mathrm{e}$ & 6 & No & Cream \\
\hline CI 12271 & Iran & 4,1460 & $68.0 \mathrm{c}$ & $41.6 f$ & $83.0 \mathrm{c}$ & $0.0707 \mathrm{~b}$ & $61.0 \mathrm{~d}$ & 6 & Yes & Cream \\
\hline MCU 3876 PI 402354 & Colombia & 5,2501 & $76.0 \mathrm{~b}$ & $52.6 \mathrm{c}$ & $85.0 \mathrm{c}$ & $0.0817 \mathrm{~b}$ & $52.6 \mathrm{~h}$ & 6 & Yes & Cream \\
\hline MCU 3827 PI 402305 & Colombia & $5,562 \mathrm{k}$ & $89.3 \mathrm{a}$ & $53.0 \mathrm{c}$ & $80.0 \mathrm{c}$ & $0.1000 \mathrm{a}$ & $50.3 \mathrm{j}$ & 6 & No & Cream \\
\hline CI 09958 & Morocco & $6,559 \mathrm{~g}$ & $93.0 \mathrm{a}$ & $47.0 \mathrm{e}$ & $82.3 \mathrm{c}$ & $0.0777 \mathrm{~b}$ & $56.0 \mathrm{~g}$ & 6 & Yes & Cream \\
\hline MCU 3469 PI 401947 & Colombia & $6,722 \mathrm{f}$ & $85.6 \mathrm{a}$ & $41.3 \mathrm{f}$ & $84.6 \mathrm{c}$ & $0.0630 \mathrm{c}$ & $49.6 \mathrm{j}$ & 6 & No & Cream \\
\hline MCU 3851 PI 402329 & Colombia & $6,962 \mathrm{e}$ & $87.6 \mathrm{a}$ & $43.3 \mathrm{f}$ & $82.3 \mathrm{c}$ & $0.1000 \mathrm{a}$ & $58.0 \mathrm{e}$ & 6 & No & Cream \\
\hline MCU 3816 PI 402294 & Colombia & $7,724 b$ & $83.3 \mathrm{a}$ & $47.3 \mathrm{e}$ & $84.6 \mathrm{c}$ & $0.1000 \mathrm{a}$ & $56.6 \mathrm{f}$ & 6 & No & Cream \\
\hline CI 13715 & Colombia & $6,303 \mathrm{~h}$ & $86.0 \mathrm{a}$ & $52.0 \mathrm{c}$ & $74.3 \mathrm{e}$ & $0.1000 \mathrm{a}$ & $55.0 \mathrm{~g}$ & 6 & No & Cream \\
\hline CI 10256 & Japan & $4,613 n$ & $81.6 \mathrm{~b}$ & $55.3 \mathrm{~b}$ & $74.0 \mathrm{e}$ & $0.1000 \mathrm{a}$ & $53.0 \mathrm{~h}$ & 6 & Yes & Cream \\
\hline CI 06946 & Iran & $5,822 \mathrm{j}$ & $79.0 \mathrm{~b}$ & $44.3 \mathrm{f}$ & $90.3 b$ & $0.1000 \mathrm{a}$ & $55.6 \mathrm{~g}$ & 6 & Yes & Cream \\
\hline CI 07255 & United States & $5,756 \mathrm{j}$ & $59.0 \mathrm{~d}$ & $46.3 \mathrm{e}$ & $103.6 \mathrm{a}$ & $0.1000 \mathrm{a}$ & $49.6 \mathrm{j}$ & 6 & No & Cream \\
\hline BRS 180 & Brazil & $7,358 \mathrm{c}$ & $88.6 \mathrm{a}$ & $42.6 \mathrm{f}$ & $85.0 \mathrm{c}$ & $0.0107 \mathrm{e}$ & $50.0 \mathrm{j}$ & 6 & Yes & Cream \\
\hline CI 15591 QB 139-1 & Canada & $7,286 \mathrm{~d}$ & $81.3 \mathrm{~b}$ & $50.3 \mathrm{~d}$ & $92.3 b$ & $0.1000 \mathrm{a}$ & $51.6 \mathrm{i}$ & 6 & No & Cream \\
\hline CI 07607 & India & $5,729 \mathrm{j}$ & $65.0 \mathrm{c}$ & $37.6 \mathrm{~g}$ & $82.3 \mathrm{c}$ & $0.1000 \mathrm{a}$ & $53.3 \mathrm{~h}$ & 6 & No & Cream \\
\hline MCU 3556 PI 402034 & Colombia & $6,336 \mathrm{~h}$ & $78.0 \mathrm{~b}$ & $42.3 \mathrm{f}$ & $89.0 \mathrm{~b}$ & $0.0000 \mathrm{f}$ & $53.3 \mathrm{~h}$ & 6 & No & Cream \\
\hline CI 07156 & United States & $7,037 \mathrm{e}$ & $71.0 \mathrm{c}$ & $46.0 \mathrm{e}$ & $84.3 \mathrm{c}$ & $0.1000 \mathrm{a}$ & $61.0 \mathrm{~d}$ & 6 & No & Cream \\
\hline CI 10078 Atlas 57 & United States & $5,670 \mathrm{j}$ & $89.0 \mathrm{a}$ & $51.0 \mathrm{c}$ & $92.0 \mathrm{~b}$ & $0.0827 \mathrm{~b}$ & $61.3 \mathrm{~d}$ & 6 & Yes & Cream \\
\hline
\end{tabular}

Continuation... 
Continuation...

\begin{tabular}{|c|c|c|c|c|c|c|c|c|c|c|}
\hline Genotype & Origin & $\begin{array}{c}\text { Yield } \\
\left(\mathrm{kg} \mathrm{ha}^{-1}\right)\end{array}$ & $\begin{array}{l}\text { PK } \\
(\%)\end{array}$ & $\begin{array}{l}\text { TSW } \\
(\mathrm{g})\end{array}$ & $\begin{array}{l}\text { Height } \\
(\mathrm{cm})\end{array}$ & $\begin{array}{c}\text { LOD } \\
\text { (arc sine) }\end{array}$ & $\begin{array}{c}\mathrm{DH} \\
\text { (days) }\end{array}$ & Type & Cultivar & Color \\
\hline CI 11493 Frugherste Stankas & Germany & 5,474 & 81.0 & 48.3 & 72.3 & 0.0440 & 57.0 & 2 & Yes & Cream \\
\hline CI 09882 Gondar & Ethiopia & $5,738 \mathrm{j}$ & $49.6 \mathrm{e}$ & $57.0 \mathrm{~b}$ & $85.6 \mathrm{c}$ & $0.0630 \mathrm{c}$ & $60.6 \mathrm{~d}$ & 6 & Yes & Cream \\
\hline CI 12598 & Ethiopia & $4,538 \mathrm{n}$ & $64.3 \mathrm{c}$ & $51.0 \mathrm{c}$ & $84.3 \mathrm{c}$ & $0.0743 b$ & $49.6 \mathrm{j}$ & 6 & Yes & Cream \\
\hline CI 10082 Weibull S 4468 & Sweden & $5,539 \mathrm{k}$ & $66.0 \mathrm{c}$ & $45.0 \mathrm{e}$ & $87.6 \mathrm{~b}$ & $0.1000 \mathrm{a}$ & $63.6 \mathrm{~b}$ & 2 & Yes & Cream \\
\hline CI 15565 QB 136-20 & Canada & $6,414 \mathrm{~g}$ & $88.3 \mathrm{a}$ & $33.0 \mathrm{~h}$ & $90.0 \mathrm{~b}$ & $0.1000 \mathrm{a}$ & $50.0 \mathrm{j}$ & 6 & No & Cream \\
\hline CI 14925 ELS 6402-512 & Ethiopia & $6,358 \mathrm{~g}$ & $75.3 b$ & $44.0 \mathrm{f}$ & $87.6 \mathrm{~b}$ & $0.1000 \mathrm{a}$ & $50.6 \mathrm{j}$ & 6 & Yes & Cream \\
\hline 2043C PI 371415 & Switzerland & $6,460 \mathrm{~g}$ & $57.0 \mathrm{~d}$ & $33.6 \mathrm{~h}$ & $79.0 \mathrm{~d}$ & $0.1000 \mathrm{a}$ & $53.0 \mathrm{~h}$ & 6 & Yes & Cream \\
\hline CI 09961 & Iran & $6,146 \mathrm{~h}$ & $88.0 \mathrm{a}$ & $43.0 \mathrm{f}$ & $90.0 \mathrm{~b}$ & $0.1000 \mathrm{a}$ & $54.0 \mathrm{~h}$ & 6 & Yes & Cream \\
\hline CI 08053 Custer & United States & $7,559 b$ & $65.0 \mathrm{c}$ & $47.6 \mathrm{e}$ & $93.6 \mathrm{a}$ & $0.1000 \mathrm{a}$ & $57.6 \mathrm{e}$ & 6 & Yes & Cream \\
\hline MCU 3461 PI 401939 & Colombia & $6,751 \mathrm{f}$ & $85.3 \mathrm{a}$ & $47.0 \mathrm{e}$ & $95.0 \mathrm{a}$ & $0.1000 \mathrm{a}$ & $51.0 \mathrm{i}$ & 6 & No & Cream \\
\hline MCU 3484 PI 401962 & Colombia & $6,930 \mathrm{e}$ & $82.3 b$ & $36.6 \mathrm{~g}$ & $88.0 \mathrm{~b}$ & $0.0917 \mathrm{a}$ & $50.3 \mathrm{j}$ & 6 & No & Cream \\
\hline CI 14031 & Ethiopia & $6,601 \mathrm{f}$ & $79.3 b$ & $51.6 \mathrm{c}$ & $92.6 b$ & $0.0777 \mathrm{~b}$ & $60.0 \mathrm{~d}$ & 6 & Yes & Cream \\
\hline CI 10017 Raspa Comun 1085 & Colombia & $5,534 \mathrm{k}$ & $86.3 \mathrm{a}$ & $51.6 \mathrm{c}$ & $83.0 \mathrm{c}$ & $0.1000 \mathrm{a}$ & $57.3 \mathrm{f}$ & 6 & Yes & Cream \\
\hline CI 15279 2528-23 & Tunisia & $6,233 \mathrm{~h}$ & $65.6 \mathrm{c}$ & $48.6 \mathrm{~d}$ & $87.6 \mathrm{~b}$ & $0.1000 \mathrm{a}$ & $55.6 \mathrm{~g}$ & 6 & Yes & Cream \\
\hline CI 15302 1356-33 & Tunisia & 5,0981 & $67.0 \mathrm{c}$ & $33.3 \mathrm{~h}$ & $91.6 \mathrm{~b}$ & $0.1000 \mathrm{a}$ & $56.0 \mathrm{~g}$ & 6 & Yes & Cream \\
\hline MCU 3454 PI 401932 & Colombia & 5,1171 & $85.3 \mathrm{a}$ & $44.6 \mathrm{e}$ & $89.3 b$ & $0.1000 \mathrm{a}$ & $50.0 \mathrm{j}$ & 6 & No & Cream \\
\hline CI 15580 QB 136-41 & Canada & $6,152 \mathrm{~h}$ & $70.3 \mathrm{c}$ & $47.0 \mathrm{e}$ & $94.0 \mathrm{a}$ & $0.0917 \mathrm{a}$ & $50.3 \mathrm{j}$ & 6 & No & Cream \\
\hline CI 07772 & India & $5,949 \mathrm{i}$ & $87.6 \mathrm{a}$ & $45.3 \mathrm{e}$ & $74.3 \mathrm{e}$ & $0.0857 \mathrm{a}$ & $55.0 \mathrm{~g}$ & 6 & Yes & Cream \\
\hline CI 14041 & Ethiopia & $5,918 \mathrm{i}$ & $78.6 \mathrm{~b}$ & $58.6 \mathrm{a}$ & $87.6 b$ & $0.0543 \mathrm{c}$ & $56.6 f$ & 6 & Yes & Cream \\
\hline MCU 3467 PI 401945 & Colombia & $4,745 \mathrm{~m}$ & $80.3 b$ & $52.0 \mathrm{c}$ & $90.6 b$ & $0.0707 \mathrm{~b}$ & $50.3 \mathrm{j}$ & 6 & No & Cream \\
\hline CI 06109 Velvon & United States & 5,1811 & $81.0 \mathrm{~b}$ & $53.0 \mathrm{c}$ & $82.3 \mathrm{c}$ & $0.0763 b$ & $60.3 \mathrm{~d}$ & 6 & Yes & Cream \\
\hline MCU 3478 PI 401956 & Colombia & $6,054 \mathrm{i}$ & $86.0 \mathrm{a}$ & $44.6 \mathrm{e}$ & $86.0 \mathrm{c}$ & $0.1000 \mathrm{a}$ & $53.0 \mathrm{~h}$ & 6 & No & Cream \\
\hline CI 09962 & Iran & $5,758 \mathrm{j}$ & $87.6 \mathrm{a}$ & $62.6 \mathrm{a}$ & $76.3 d$ & $0.0817 b$ & $56.0 \mathrm{~g}$ & 6 & Yes & Cream \\
\hline MCU 3878 PI 402356 & Colombia & $6,233 \mathrm{~h}$ & $77.0 \mathrm{~b}$ & $51.3 \mathrm{c}$ & $84.6 \mathrm{c}$ & $0.0683 b$ & $50.0 \mathrm{j}$ & 6 & No & Cream \\
\hline CI 10697 & Iran & $4,740 \mathrm{~m}$ & $71.3 \mathrm{c}$ & $53.6 \mathrm{c}$ & $84.6 \mathrm{c}$ & $0.1000 \mathrm{a}$ & $50.3 \mathrm{j}$ & 6 & Yes & Cream \\
\hline CI $153232222-79$ & Tunisia & $5,735 \mathrm{j}$ & $84.6 \mathrm{a}$ & $55.3 \mathrm{~b}$ & $85.6 \mathrm{c}$ & $0.1000 \mathrm{a}$ & $57.6 \mathrm{e}$ & 6 & Yes & Cream \\
\hline MCU 3750 PI 402228 & Colombia & 5,2051 & $84.3 \mathrm{a}$ & $54.3 \mathrm{c}$ & $85.6 \mathrm{c}$ & $0.0577 \mathrm{c}$ & $58.3 \mathrm{e}$ & 6 & No & Cream \\
\hline CI 12918 & Ethiopia & $7,207 d$ & $77.0 \mathrm{~b}$ & $60.3 \mathrm{a}$ & $87.6 \mathrm{~b}$ & $0.1000 \mathrm{a}$ & $57.0 \mathrm{f}$ & 6 & Yes & Cream \\
\hline MCU 3634 PI 402112 & Colombia & $7,132 d$ & $87.3 \mathrm{a}$ & $46.6 \mathrm{e}$ & $82.0 \mathrm{c}$ & $0.1000 \mathrm{a}$ & $51.0 \mathrm{i}$ & 6 & No & Cream \\
\hline E 273/4 PI 356414 & Ethiopia & $6,128 \mathrm{~h}$ & $46.0 \mathrm{e}$ & $52.0 \mathrm{c}$ & $76.6 \mathrm{~d}$ & $0.0707 \mathrm{~b}$ & $52.0 \mathrm{i}$ & 6 & Yes & Cream \\
\hline CARINA PI 371632 & Germany & $6,225 \mathrm{~h}$ & $75.0 \mathrm{~b}$ & $49.3 \mathrm{~d}$ & $79.0 \mathrm{~d}$ & $0.0107 \mathrm{e}$ & $62.0 \mathrm{c}$ & 2 & Yes & Cream \\
\hline CI 15281 3102-16 & Tunisia & 4,493 & 58.0 & 45.6 & 92.0 & 0.1000 & 68.0 & $2 / 6$ & Yes & Cream \\
\hline CI 02109 White Smyrna & United States & 5,0541 & $66.6 \mathrm{c}$ & $54.6 \mathrm{c}$ & $87.6 b$ & $0.1000 \mathrm{a}$ & $60.0 \mathrm{~d}$ & 2 & Yes & Cream \\
\hline MCU 3865 PI 402343 & Colombia & $7,215 \mathrm{~d}$ & $85.6 \mathrm{a}$ & $53.6 \mathrm{c}$ & $83.0 \mathrm{c}$ & $0.0707 \mathrm{~b}$ & $58.0 \mathrm{e}$ & 6 & No & Cream \\
\hline CI 13704 & England & 4,1520 & $80.6 \mathrm{~b}$ & $56.0 \mathrm{~b}$ & $90.0 \mathrm{~b}$ & $0.0733 b$ & $63.0 \mathrm{~b}$ & 2 & Yes & Black \\
\hline CI 12367 Branisovicky & Czech Republic & $5,447 \mathrm{k}$ & $89.6 \mathrm{a}$ & $35.3 \mathrm{~g}$ & $84.0 \mathrm{c}$ & $0.0000 \mathrm{f}$ & $67.3 \mathrm{a}$ & 2 & Yes & Cream \\
\hline CI 12262 & Iran & $4,249 \mathrm{o}$ & $79.6 \mathrm{~b}$ & $35.6 \mathrm{~g}$ & $85.0 \mathrm{c}$ & $0.1000 \mathrm{a}$ & $68.3 \mathrm{a}$ & 6 & Yes & Cream \\
\hline CI 10114 Carlsberg II & Denmark & $4,737 \mathrm{~m}$ & $88.3 \mathrm{a}$ & $45.0 \mathrm{e}$ & $83.3 \mathrm{c}$ & $0.0757 \mathrm{~b}$ & $68.0 \mathrm{a}$ & 2 & Yes & Cream \\
\hline MCU 3852 PI 402330 & Colombia & $7,360 \mathrm{c}$ & $88.0 \mathrm{a}$ & $47.0 \mathrm{e}$ & $80.6 \mathrm{c}$ & $0.0440 \mathrm{~d}$ & $57.3 \mathrm{f}$ & 6 & No & Cream \\
\hline A HOR 55/61 PI 327910 & Bulgaria & $6,124 \mathrm{~h}$ & $60.3 \mathrm{~d}$ & $44.0 \mathrm{f}$ & $79.0 \mathrm{~d}$ & $0.1000 \mathrm{a}$ & $56.3 f$ & 6 & Yes & Cream \\
\hline CI 10140 & Afghanistan & $4,307 \mathrm{o}$ & $80.6 \mathrm{~b}$ & $46.6 \mathrm{e}$ & $87.6 \mathrm{~b}$ & $0.0777 \mathrm{~b}$ & $53.0 \mathrm{~h}$ & 6 & Yes & Cream \\
\hline MCU 3884 PI 402362 & Colombia & $6,226 \mathrm{~h}$ & $84.6 \mathrm{a}$ & $46.6 \mathrm{e}$ & $73.3 \mathrm{e}$ & $0.1000 \mathrm{a}$ & $50.3 \mathrm{j}$ & 6 & No & Cream \\
\hline MCU 3778 PI 402256 & Colombia & $4,424 n$ & $73.6 \mathrm{~b}$ & $44.6 \mathrm{e}$ & $95.6 \mathrm{a}$ & $0.1000 \mathrm{a}$ & $55.0 \mathrm{~g}$ & 6 & Yes & Cream \\
\hline CI 09952 & Russia & $6,441 \mathrm{~g}$ & $85.0 \mathrm{a}$ & $48.3 \mathrm{~d}$ & $87.6 \mathrm{~b}$ & $0.0950 \mathrm{a}$ & $55.3 \mathrm{~g}$ & 6 & Yes & Cream \\
\hline MCU 3448 PI 401926 & Colombia & $5,757 \mathrm{j}$ & $71.6 \mathrm{c}$ & $37.3 \mathrm{~g}$ & $92.3 b$ & $0.0543 \mathrm{c}$ & $53.0 \mathrm{~h}$ & 6 & No & Cream \\
\hline CI 06244 & United States & $5,883 \mathrm{i}$ & $84.6 \mathrm{a}$ & $45.0 \mathrm{e}$ & $84.3 \mathrm{c}$ & $0.1000 \mathrm{a}$ & $52.6 \mathrm{~h}$ & 6 & Yes & Cream \\
\hline MCU 3449 PI 401927 & Colombia & $6,818 \mathrm{e}$ & $90.3 \mathrm{a}$ & $51.3 \mathrm{c}$ & $84.6 \mathrm{c}$ & $0.0000 \mathrm{f}$ & $50.3 \mathrm{j}$ & 6 & No & Cream \\
\hline MCU 3654 PI 402132 & Colombia & $7,182 \mathrm{~d}$ & $90.6 \mathrm{a}$ & $46.3 \mathrm{e}$ & $86.6 \mathrm{~b}$ & $0.1000 \mathrm{a}$ & $50.6 \mathrm{j}$ & 6 & No & Cream \\
\hline CI 12068 Mazowiecki & Poland & $6,740 \mathrm{f}$ & $72.3 \mathrm{c}$ & $41.6 f$ & $86.6 b$ & $0.1000 \mathrm{a}$ & $50.6 \mathrm{j}$ & 6 & Yes & Cream \\
\hline MCU 3502 PI 401980 & Colombia & $6,170 \mathrm{~h}$ & $92.0 \mathrm{a}$ & $45.6 \mathrm{e}$ & $90.6 b$ & $0.1000 \mathrm{a}$ & $50.6 \mathrm{j}$ & 6 & No & Cream \\
\hline MCU 3870 PI 402348 & Colombia & $7,347 \mathrm{c}$ & $81.0 \mathrm{~b}$ & $42.0 \mathrm{f}$ & $97.6 \mathrm{a}$ & $0.1000 \mathrm{a}$ & $50.3 \mathrm{j}$ & 6 & No & Cream \\
\hline CI 10071 Wolfe & Canada & $6,068 \mathrm{i}$ & $81.0 \mathrm{~b}$ & $36.6 \mathrm{~g}$ & $95.0 \mathrm{a}$ & $0.1000 \mathrm{a}$ & $50.3 \mathrm{j}$ & 6 & Yes & Cream \\
\hline CI 13711 & Colombia & $5,690 \mathrm{j}$ & $79.3 b$ & $44.0 \mathrm{f}$ & $87.6 \mathrm{~b}$ & $0.1000 \mathrm{a}$ & $49.3 \mathrm{j}$ & 6 & No & Cream \\
\hline CI 10022 & Colombia & $6,434 \mathrm{~g}$ & $75.6 \mathrm{~b}$ & $36.6 \mathrm{~g}$ & $82.3 \mathrm{c}$ & $0.1000 \mathrm{a}$ & $52.0 \mathrm{i}$ & 6 & Yes & Cream \\
\hline CI 13824 Atlas 68 & United States & $6,738 \mathrm{f}$ & $74.6 \mathrm{~b}$ & $52.6 \mathrm{c}$ & $83.6 \mathrm{c}$ & $0.1000 \mathrm{a}$ & $53.3 \mathrm{~h}$ & 6 & Yes & Cream \\
\hline MCU 3850 PI 402328 & Colombia & $5,979 \mathrm{i}$ & $72.6 \mathrm{c}$ & $51.6 \mathrm{c}$ & $87.6 \mathrm{~b}$ & $0.0897 \mathrm{a}$ & $58.6 \mathrm{e}$ & 6 & Yes & Cream \\
\hline
\end{tabular}

(1)Yield, grain yield; PK, kernel plumpness; TSW, thousand seed weight; Height, plant height; LOD, lodging; and DH, days to heading. Different letters next to treatments indicate significant differences by the Scott-Knott test, at $1 \%$ probability. 
the respective variances and covariances between the traits, two by two, according to Kempthorne (1966), also using the Genes software (Universidade Federal de Viçosa, Viçosa, MG, Brazil).

For the classification of the correlations, the intervals proposed by Carvalho et al. (2004) were adopted, in which the intensities are ranked as: perfect $(|\mathrm{r}|=1)$; very strong $(0.90 \leq|\mathrm{r}|<1.00)$; strong $(0.60 \leq|\mathrm{r}|<0.90)$; medium $(0.30 \leq|\mathrm{r}|<0.60)$; weak $(0.00<|\mathrm{r}|<0.30)$; and null $(\mathrm{r}=0)$.

\section{Results and Discussion}

The joint Anova showed the existence of significant differences among the accessions for all evaluated traits (Table 3), highlighting the variability among barley accessions, which is desired in pre-breeding works.

Significant effects of environment and genotype $x$ environment interaction were also observed for the studied traits. This can be largely attributed to the difference in altitude between the experimental fields, of approximately $200 \mathrm{~m}$, which directly affects temperature and favors environments of milder climates. This difference contributes to the increase in the genotype $\mathrm{x}$ environment interaction and, consequently, to the greater phenotypic variability of the quantitative traits, as reported by Kaczmarek et al. (1999).

According to Resende \& Duarte (2007), regarding selective accuracy, in order to achieve values of $90 \%$ or more, considered ideal by Steel \& Torrie (1980), F-values for the cultivar must be equal to or higher than 5.26 for a safe statistical interference. In the present study, regarding genotype $\mathrm{x}$ environment interaction, the F-value was only lower than the ones proposed by Resende \& Duarte (2007) for lodging (4.7), showing high selection accuracy (Table 3 ).

Another genetic parameter that indicates experimental precision is the EVC; the lower this value is, the more precise is the experiment. However, the analysis of the EVC value must be considered according to the particularities of each trait and crop evaluated (Costa et al., 2002). In the present study, the values of the EVC varied from $1.39 \%$ for $\mathrm{DH}$ to $6.93 \%$ for LOD, which are considered low, indicating a good environment control (Table 3).
Genotypic variance is associated with the genetic differences among the specimens. Higher values for this component are an indication of higher genetic variability, which enables the identification of superior genotypes (Vencovsky, 1987). It was observed that the estimate of genotypic variance was the main component of phenotypic variance among the following traits: yield, kernel plumpness, TSW, height, LOD, and DH (Table 3).

The $\mathrm{CVr}$ is represented by the ratio between the GVC and the EVC. According to Vencovsky (1987), values above the unit for the CVr indicate good chances of gains with the application of selection among populations, suggesting that the trait can be handled with higher probability of genetic gains in breeding in experiments with two or three replicates.

The values of the GVC were higher than those of the EVC for all traits. The traits with higher $\mathrm{CVr}$ were: DH, yield, and LOD, with 6.23, 4.55, and 3.88\%, respectively (Table 3 ). The value of the $\mathrm{CVr}$ for $\mathrm{LOD}$ contrasts with that obtained by Amabile et al. (2015) in irrigated conditions in the Cerrado, which can be explained by the fact that these authors studied a lower number of cultivars, which are considered elite and, therefore, probably presented a lower $\mathrm{CVr}$ value for LOD (0.54\%); however, as in the present study (Table 3), Amabile et al. (2015) also found high values for $\mathrm{DH}$ (5.33\%) and yield (2.72\%).

The percentage of genetic variability that is transmitted from the parents to the descendants is obtained through $\mathrm{h}^{2}$ (Lush, 1949). The high yields aspired by the breeder can be reached by the selection of traits that contribute to grain yield and that have both high $\mathrm{h}^{2}$ and genetic gain. Consequently, the components of variance and $\mathrm{h}^{2}$ with the genetic parameters are important for the definition of strategies aiming at the higher efficiency of the breeding program. The broadsense $\mathrm{h}^{2}$ ranged from $99.57 \%$ for LOD to $97.91 \%$ for TSW (Table 3). The following values of high magnitude were also found for the same traits: above $84 \%$, in 18 accessions gathered in Ethiopia (Addisu \& Shumet, 2015); and from 62 to $84 \%$, in F1 and F2 populations from ten Indian cultivars in a study performed during winter in India (Yadav et al., 2015).

The genotypic, phenotypic, and residual correlation coefficients between the evaluated traits in the present study were, in general, considered of low magnitude when compared with those found by Sayd (2014) and 
Amabile et al. (2015), of 0.6 and 0.8, respectively. However, higher relationships, of 0.4 , were observed between PK and TSW (Table 4), which is in alignment with Amabile et al. (2015), who reported a strong correlation of 0.6 between PK and TSW, which facilitates the evaluation process of the traits and also indirect selection. The genotypic and phenotypic correlation coefficients between height and LOD, in turn, were inexpressive, allowing the selection of tall plants with an adequate architecture that minimizes lodging, according to Ceccarelli et al. (1992).

In the comparison between averages for estimated grain yield at the CPAC site, the Colombian genotype MCU 3484 PI 401962 stood out positively with $6,508 \mathrm{~kg} \mathrm{ha}^{-1}$, similarly to the Brazilian control cultivar BRS 180 , with $6,142 \mathrm{~kg} \mathrm{ha}^{-1}$ (Table 1). At the SPM site (Table 2), the control cultivar BRS 180, with $7,358 \mathrm{~kg} \mathrm{ha}^{-1}$, was overcome statistically by the four following genotypes, presenting values considered of high magnitude: the Afghan cultivar H HOR 2325/58 PI 329126, with 9,187 $\mathrm{kg} \mathrm{ha}^{-1}$; the Colombian genotypes MCU 3816 PI 402294 and MCU 3832 PI 402310, with 7,724 and 7,695 $\mathrm{kg} \mathrm{ha}^{-1}$, respectively; and the North American cultivar CI 08053 Custer, with 7,559 $\mathrm{kg} \mathrm{ha}^{-1}$ (Table 2). At both sites, phenotypes from the American continent presented higher yield potential when compared with the genotypes from the other continents. Moreover, as expected, the six-row genotypes showed higher values for yield.
Values of $9,000 \mathrm{~kg} \mathrm{ha}^{-1}$ were also achieved in experiments at Embrapa Cerrados with lineages from International Maize and Wheat Improvement Center (CIMMYT) introduced into the Cerrado, but with no malt quality (Amabile \& Faleiro, 2014). Sanchez et al. (2015) obtained lower values - 5,848, 5,449, and 4,767 $\mathrm{kg} \mathrm{ha}^{-1}$, respectively, in the Cerrado - for the BRS Sampa, Manduri, and BRS 195 cultivars, recommended for irrigated areas. Values lower than the ones observed in the present study were also reported by Amabile et al. (2013), who found average of $5,908 \mathrm{~kg} \mathrm{ha}^{-1}$ for the BRS Savanna cultivar, with values ranging from 4,726 to $8,659 \mathrm{~kg} \mathrm{ha}^{-1}$ in trials performed in different locations and years in the Cerrado. However, there is still the possibility of grain yield gains using genotypes from the germplasm bank that are being introduced as cultivars and used in breeding blocks.

For kernel plumpness, the SPM site presented 51 genotypes with values above $80 \%$, the lower limit adopted by the breeding program, whereas the CPAC site presented only 13 genotypes. At the CPAC site, the following genotypes stood out positively: six-row cultivars from Morocco - CI 09958 (87.6\%) and CI 09959 (84.6\%) - and from Iran - CI 09962 (91.6\%) and CI 09961 (85.3\%); besides two-row cultivars from Germany - CI 11493 Frugherste Stankas (82\%) and Carina PI 371632 (84\%) - and from the Czech Republic - CI 11367 Branisovicky (81\%) (Table 4). At the SPM site, 37 genotypes were similar to the control BRS 180

Table 3. Analysis of variance and genetic parameters for grain yield, kernel plumpness (PK), thousand seed weight (TSW), plant height, degree of plant lodging (LOD), and days to heading (DH), evaluated in 113 barley (Hordeum vulgare) accessions at two sites.

\begin{tabular}{|c|c|c|c|c|c|c|c|}
\hline \multirow[t]{2}{*}{ Sources of variation ${ }^{(1)}$} & \multirow[t]{2}{*}{$\mathrm{DF}^{(2)}$} & \multicolumn{6}{|c|}{ F-values } \\
\hline & & Yield & PK & TSW & Height & LOD & $\mathrm{DH}$ \\
\hline Blocks/Environment & 4 & & & & & & \\
\hline Blocks & 2 & & & & & & \\
\hline Block x environment & 2 & & & & & & \\
\hline Genotype & 112 & $125.7 * *$ & $69.0 * *$ & $48.0^{* *}$ & $91.5^{* *}$ & $234.4^{* *}$ & $171.2^{* *}$ \\
\hline Environment & 1 & $15,887.0^{* *}$ & $1,238.9 * *$ & $627.5^{* *}$ & $198.3^{* *}$ & $64.4 * *$ & $3,797.5^{* *}$ \\
\hline Genotype x Environment & 112 & $55.7 * *$ & $19.7 * *$ & $18.7 * *$ & $27.0^{* *}$ & $4.7^{* *}$ & $53.5^{* *}$ \\
\hline Residue & 448 & & & & & & \\
\hline $\mathrm{h}^{2}(\%)$ & & 99.20 & 98.55 & 97.91 & 98.90 & 99.57 & 99.41 \\
\hline GVC & & 14.70 & 19.47 & 11.20 & 10.14 & 43.18 & 7.42 \\
\hline EVC & & 3.23 & 5.79 & 4.00 & 2.61 & 6.93 & 1.39 \\
\hline $\mathrm{CVr}$ & & 4.55 & 3.36 & 1.74 & 2.80 & 3.88 & 6.23 \\
\hline
\end{tabular}

${ }^{(1)} \mathrm{h}^{2}$, heritability; GVC, coefficient of genetic variance; EVC, coefficient of environmental variance; and $\mathrm{CVr}$, coefficient of relative variance. (2) DF, degrees of freedom. **Significant by the F-test, at $1 \%$ probability. 
(88.6\%), among them: six-row genotypes from Iran, Morocco, the United States, Colombia, Cyprus, Canada, India, Russia, and Tunisia; and two-row genotypes from the Czech Republic and Denmark (Table 2).

These results highlight the varying responses of the genotypes to the different evaluated environments. However, the Moroccan cultivar CI 09958 stood out at both sites, with values of $87.6 \%$ for CPAC and of $93 \%$ for SPM, showing high potential and higher stability for this trait in comparison with the other accessions. High values for kernel plumpness, above $80 \%$, were also reported by: Amabile et al. (2013), who studied 39 elite two-row and six-row genotypes in irrigated conditions in the Cerrado; and Smith et al. (2013), who assessed 7 cultivars of high quality adapted the central north region of the United States, during six years, at four different sites.

The average for TSW of the accessions at the CPAC site $(40.2 \mathrm{~g})$ was lower than the one at SPM (46.4 g), showing the difference between the two environments (Tables 1 and 2, respectively). However, the Iranian cultivar CI 09962 had high values at both sites: $57 \mathrm{~g}$ at CPAC, which was statistically similar to the weight of the Moroccan cultivar CI 09958, of $58 \mathrm{~g}$; and $62.6 \mathrm{~g}$ at SPM, which does not differ statistically from the weights of the Ethiopian cultivars CI 12918 and CI

Table 4. Genotypic $\left(\mathrm{r}_{\mathrm{g}}\right)$, phenotypic $\left(\mathrm{r}_{\mathrm{p}}\right)$, and environmental $\left(r_{e}\right)$ correlation coefficients between grain yield, kernel plumpness (PK), thousand seed weight (TSW), plant height, degree of plant lodging (LOD), and days to heading (DH) in 113 barley (Hordeum vulgare) accessions.

\begin{tabular}{lcccccc}
\hline & & Yield & PK & TSW & Height & LOD \\
\hline \multirow{2}{*}{ PK } & $\mathrm{r}_{\mathrm{p}}$ & 0.2858 & & & & \\
& $\mathrm{r}_{\mathrm{g}}$ & 0.2889 & & & & \\
& $\mathrm{r}_{\mathrm{e}}$ & 0.0166 & & & & \\
TSW & $\mathrm{r}_{\mathrm{p}}$ & -0.0739 & 0.3974 & & & \\
& $\mathrm{r}_{\mathrm{g}}$ & -0.0753 & 0.4028 & & & \\
& $\mathrm{r}_{\mathrm{e}}$ & 0.0200 & 0.1000 & & & \\
Height & $\mathrm{r}_{\mathrm{p}}$ & -0.0776 & -0.1263 & -0.1719 & & \\
& $\mathrm{r}_{\mathrm{g}}$ & -0.0790 & -0.1290 & -0.1747 & & \\
\hline \multirow{4}{*}{ LOD } & $\mathrm{r}_{\mathrm{e}}$ & 0.0637 & 0.0823 & 0.0029 & & \\
& $\mathrm{r}_{\mathrm{p}}$ & -0.0249 & -0.0241 & 0.1115 & 0.0910 & \\
& $\mathrm{r}_{\mathrm{g}}$ & -0.0245 & -0.0241 & 0.1127 & 0.0916 & \\
& $\mathrm{r}_{\mathrm{e}}$ & -0.0970 & -0.0284 & 0.0214 & 0.0038 & \\
\multirow{2}{*}{ DH } & $\mathrm{r}_{\mathrm{p}}$ & -0.3365 & -0.0139 & 0.1053 & 0.1013 & -0.0153 \\
& $\mathrm{r}_{\mathrm{g}}$ & -0.3393 & -0.0141 & 0.1064 & 0.1018 & -0.0152 \\
& $\mathrm{r}_{\mathrm{e}}$ & 0.0618 & 0.0057 & 0.0289 & 0.0402 & -0.0288 \\
\hline
\end{tabular}

14041, of 60.3 and $58.6 \mathrm{~g}$, respectively. These values show the positive correlation between the traits PK and TSW (Table 2), which is benefic to the breeding program. Among the accessions, only CI 12918 presented yield above $6,000 \mathrm{~kg} \mathrm{ha}^{-1}$ in at least one site, emphasizing the low correlation between TSW and yield. Two other two-row cultivars stood out positively at both sites: CI 02109 White Smyrna from the United States and CI 09882 Gondar from England.

In the literature, Smith et al. (2013) observed TSW ranging from 56 to $59 \mathrm{~g}$ in seven beer cultivars recently released, values close to the ones of the accessions analyzed in the present study. Mirosavljević et al. (2015) reported an average of $40.8 \mathrm{~g}$ for 19 genotypes of two-row winter beer barley during three years of assessment in Serbia.

For plant height, the tallest accessions, all above $94 \mathrm{~cm}$, were: CI 07255 from the United States and CI 06147 from Egypt (Table 1) in the CPAC site; and CI 10016 Raspa from Colombia and CI 07255 from the United States in the SPM site (Table 2). The Ethiopian cultivar E 3/416 PI 356495 showed the lowest height at both environments, $57.6 \mathrm{~cm}$ at CPAC and $69 \mathrm{~cm}$ at SPM. The small size of this genotype makes it interesting to explore, since breeding programs have predicted, among their goals, the selection of short plants to avoid lodging (Amabile et al., 2015). The height of the accessions ranged from $57.6 \mathrm{~cm}$ for $\mathrm{E}$ 3/416 PI 356495 to $99 \mathrm{~cm}$ for CI 07255 at the CPAC site, and from $69 \mathrm{~cm}$ for E 3/416 PI 356495 to $103.6 \mathrm{~cm}$ for CI 07255 at the SPM site, with averages of 79.5 and $86.4 \mathrm{~cm}$, respectively.

The Canadian genotypes presented heights above $84 \mathrm{~cm}$ at both sites and were characterized as tall. In contrast, the genotypes from South America showed great variation in height, varying from short, to medium, to tall. The Asian, European, and African genotypes showed smaller variations for this trait and were characterized as medium, except the CI 06147 cultivar from Egypt.

The behavior of the genotypes regarding lodging was considerably more intense at the SPM site than at CPAC. This behavior is due to the higher altitude of the first environment, which creates a more humid microclimate, favoring higher growth and, consequently, plant lodging. Of the 113 accessions evaluated, 14 did not lodge at the CPAC site and 8 did not lodge at the SPM site. Still, at the SPM site, 87 
genotypes presented high lodging, above 0.0630 , while at CPAC, 70 genotypes showed high lodging.

The values for lodging obtained in the present study were higher than the averages of $28 \%$ reported by Mirosavljević et al. (2015), in trials performed with winter barley during three years, and of $25 \%$, by Smith et al. (2013), for seven cultivars released in the United States. The Colombian genotypes MCU 3592 PI 402070 , MCU 3576 PI 402054, and MCU 3452 PI 401930, as well as the control cultivar BRS 180 , stood out positively and did not present lodging at both environments. No grouping tendency was observed when considering countries of origin and level of lodging.

Lodging, therefore, is a non-limiting trait, which can be solved with the use of growth reducers. However, the search for high-yielding genotypes with a more resistant stem allows the producer to reduce production costs and diminishes the risk of losses at harvest due to lodging.

Regarding $\mathrm{DH}$, the most precocious genotypes were all six-row, originating from Colombia: MCU 3571 PI 402049, CI 13711 (both 49.3 days), and MCU 3469 PI 401947 (49.6 days) at the SPM site; and MCU 3484 PI 401962 (50.3 days) and MCU 3827 PI 402305 (51 days) at the CPAC site. The genotypes with higher yields presented heading cycles of around 50 to 60 days, similar to the ones obtained by the cultivar recommended for the Cerrado by Amabile et al. (2013). Values of around 55 days were also found for seven cultivars released in the United States in trials performed during six years by Smith et al. (2013).

The average of the genotypes was of 55 days at the SPM site and of 61 days at the CPAC site, with a difference of 6 days from one environment to the other (Tables 1 and 2). These averages are considered low when compared with the one obtained by Yadav et al. (2015) of 75 days for precocious Indian genotypes, in trials performed in India. It should be noted that more precocious genotypes are interesting for use in irrigated systems in Brazil, considering the advantage of the early release of areas for sowing of summer crops in the country.

\section{Conclusions}

1. The MCU 3832 PI 402310, MCU 3484 PI 401962 , CI 08053 Custer, MCU 3634 PI 402112, and MCU 3816 PI 402294 accessions stand out regarding grain yield and kernel plumpness.
2. High selection gains may be obtained with high hereditability values, but indirect selection is not recommended in the experimental conditions because of the low genotypic and phenotypic correlations observed.

3. It is possible to select barley accessions with high yield and precocious heading cycle simultaneously, a decisive factor in the process of genotype selection for future trials.

4. The accessions from Colombia are the most adapted to the Cerrado conditions.

\section{Acknowledgments}

To Coordenação de Aperfeiçoamento de Pessoal de Nível Superior (Capes), for the scholarship granted.

\section{References}

ADDISU, A.; SHUMET, T. Variability, heritability and genetic advance for some yield and yield related traits in barley (Hordeum vulgare L.) landraces in Ethiopia. International Journal of Plant Breeding and Genetics, v.9, p.68-76, 2015. DOI: 10.3923/ ijpbg.2015.68.76.

AGOSTINETTO, L.; CASA, R.T.; BOGO, A.; SACHS, C.; SOUZA, C.A.; REIS, E.M.; CUNHA, I.C. da. Barley spot blotch intensity, damage, and control response to foliar fungicide application in southern Brazil. Crop Protection, v.67, p.7-12, 2015. DOI: 10.1016/j.cropro.2014.09.012.

AMABILE, R.F.; CAPETTINI, F.; FALEIRO, F.G. BRS Savanna: new six-rowed malting barley cultivar for irrigated crops in the Brazilian savanna. Crop Breeding and Applied Biotechnology, v.13, p.160-163, 2013.

AMABILE, R.F.; FALEIRO, F.G. A cevada irrigada no Cerrado: estado da arte, recursos genéticos e melhoramento. 2014. 127p.

AMABILE, R.F.; FALEIRO, F.G.; CAPETTINI, F.; PEIXOTO, J.R.; SAYD, R. Estimation of genetic parameters, phenotypic, genotypic and environmental correlations on barley (Hordeum vulgare L.) grown under irrigation conditions in the Brazilian savanna. Interciencia, v.40, p.255-262, 2015.

AMABILE, R.F.; FALEIRO, F.G.; CAPETTINI, F.; SAYD, R.M.; PEIXOTO, J.R.; GUERCIA, R.F. Characterization and genetic variability of barley accessions (Hordeum vulgare L.) irrigated in the savannas based on malting quality traits. Journal of the Institute of Brewing, v.120, p.404-414, 2014. DOI: 10.1002/ jib.179.

AMABILE, R.F.; MINELLA, E.; OLIVEIRA, C.M. de; FRONZA, V. Cevada (Hordeum vulgare L.). In: PAULA JÚNIOR, T.J. de; VENZON, M. (Coord.). 101 culturas: manual de tecnologias agrícolas. Belo Horizonte: Epamig, 2007. p.263-268.

BRASIL. Ministério da Agricultura e Abastecimento. Portaria $n^{\circ}$ 691, de 22 de novembro de 1996. Aprova a Norma de identidade 
e qualidade da cevada, para comercialização. Diário Oficial [da] República Federativa do Brasil, 25 nov. 1996. Seção 1, p.2475124752.

CARVALHO, F.I.F. de; LORENCETTI, C.; BENIN, G. Estimativas e implicações da correlação: no melhoramento vegetal. Pelotas: Ed. da UFPel, 2004. 142p.

CECCARELLI, S.; GRANDO, S.; HAMBLIN, J. Relationship between barley grain yield measured in low- and high-yielding environments. Euphytica, v.64, p.49-58, 1992. DOI: 10.1007/ BF00023537.

COSTA, N.H.A. de D.; SERAPHIN, J.C.; ZIMMERMANN, F.J.P. Novo método de classificação de coeficientes de variação para a cultura do arroz de terras altas. Pesquisa Agropecuária Brasileira, v.37, p.243-249, 2002. DOI: 10.1590/S0100204X2002000300003.

FAOSTAT. Statistical databases. Available at: <http://faostat. fao.org>. Accessed on: Jul. 142016.

FERREIRA, J.R.; PEREIRA, J.F.; TURCHETTO, C.; MINELLA, E.; CONSOLI, L.; DELATORRE, C.A. Assessment of genetic diversity in Brazilian barley using SSR markers. Genetics and Molecular Biology, v.39, p.86-96, 2016. DOI: 10.1590/1678-4685GMB-2015-0148.

KACZMAREK, J.; ADAMSKI, T.; SURMA, M.; JEŻOWSKI, S.; LEŚNIEWSKA-FRATCZAK, M. Genotype-environment interaction of barley double haploids with regard to malting quality. Plant Breeding, v.118, p.243-247, 1999. DOI: 10.1046/j.14390523.1999.118003243.x.

KEMPTHORNE, O. An introduction to genetic statistics. New York: Wiley, 1966. 545p.

LUSH, J.L. Heritability of quantitative traits in farm animals. Proceedings of the 8th International Congress on Genetics, v.35, p.356-375, 1949. Supplement.

MINELLA, E. Barley in tropical areas: the Brazilian experience. In: ZHANG, G.; LI, C.; LIU, X. (Ed.). Advance in barley sciences. Dordrecht: Springer, 2013. p.359-366. DOI: 10.1007/978-94-0074682-4_30.

MIROSAVLJEVIĆ, $\quad$ M.; PRŽULJ, N.; ČANAK, P.; MOMČILOVIĆ, V.; AĆIN, V.; JOCKOVIĆ, B.; HRISTOV,
N.; MLADENOV, N. Relationship between grain yield and agronomic traits in winter barley. Ratarstvo i Povrtarstvo, v.52, p.74-79, 2015. DOI: 10.5937/ratpov52-7860.

MONTEIRO, V.A. Diversidade genética de acessos de cevada sob sistema de produção irrigado no Cerrado do Planalto Central brasileiro. 2012. 136p. Dissertação (Mestrado) Universidade de Brasília, Brasília.

REGRAS para análise de sementes. Brasília: Ministério da Agricultura, Pecuária e Abastecimento, 2009. 395p.

RESENDE, M.D.V. de; DUARTE, J.B. Precisão e controle de qualidade em experimentos de avaliação de cultivares. Pesquisa Agropecuária Tropical, v.37, p.182-194, 2007.

SANCHEZ, F.M.; CUNHA, F.F. da; SANTOS, O.F. dos; SOUZA, E.J. de; LEAL, A.J.F.; THEODORO, G. de F. Desempenho agronômico de cultivares de cevada cervejeira sob diferentes lâminas de irrigação. Semina: Ciências Agrárias, v.36, p.89-102, 2015. DOI: 10.5433/1679-0359.2015v36n1p89.

SAYD, R.M. Variabilidade, parâmetros genéticos e caracterização agronômica e molecular de genótipos de cevada nua (Hordeum vulgare l. Var. nudum hook. F.) sob irrigação no Cerrado. 2014. 83p. Dissertação (Mestrado em Agronomia) Universidade de Brasília, Brasília.

SMITH, K.P.; BUDDE, A.; DILL-MACKY, R.; RASMUSSON, D.C.; SCHIEFELBEIN, E.; STEFFENSON, B.; WIERSMA, J.J.; WIERSMA, J.V.; ZHANG, B. Registration of 'Quest' spring malting barley with improved resistance to Fusarium head blight. Journal of Plant Registrations, v.7, p.125-129, 2013. DOI: 10.3198/jpr2012.03.0200crc.

STEEL, R.G.D.; TORRIE, J.H. Principles and procedures of statistics: a biometrical approach. $2^{\text {nd }}$ ed. New York: McGrawHill, 1980. 633p.

VENCOVSKY, R. Herança quantitativa. In: PATERNIANI, E.; VIEGAS, G.P. (Ed.). Melhoramento e produção de milho. 2.ed. Campinas: Fundação Cargill, 1987. p.122-201.

YADAV, S.K.; SINGH, A.K.; PANDEY, P.; SINGH, S. Genetic variability and direct selection criterion for seed yield in segregating generations of barley (Hordeum vulgare L.). American Journal of Plant Sciences, v.6, p.1543-1549, 2015. DOI: 10.4236/ajps.2015.69153.

Received on May 27, 2016 and accepted on August 4, 2016

Pesq. agropec. bras., Brasília, v.52, n.2, p.84-94, fev. 2017

DOI: $10.1590 / \mathrm{S} 0100-204 X 2017000200002$ 